\title{
OPTIMAL AMPLIFY-AND-FORWARD PRECODE AND RELAY AMPLIFYING MATRICES
}

\author{
A Thesis by \\ Hyunggi Kim \\ Bachelor of Science, Republic Of Korea Air force Academy, 2000
}

Submitted to the Department of Electrical and Computer Engineering and the faculty of the Graduate School of

Wichita State University

in partial fulfillment of

the requirements for the degree of

Master of Science 
July 2013

(C) Copyright 2013 by Hyunggi Kim

All Rights Reserved

Note that thesis work is protected by copyright, with all rights reserved. Only the author and advisor Dr. Hyuck M. Kwon have the legal right to publish, produce, sell, or distribute this work. Author or advisor permission is needed for others to directly quote significant amounts of information in their own work or to summarize substantial amounts of information in their own work. Limited amounts of information cited, paraphrased, or summarized from the work may be used with proper citation of where to find the original work. 


\section{OPTIMAL AMPLIFY-AND-FORWARD PRECODE AND RELAY AMPLIFYING MATRICES}

The following faculty members have examined the final copy of this thesis for form and content, and recommend that it be accepted in partial fulfillment of the requirement for the degree of Master of Science with a major in Electrical Engineering.

Hyuck M. Kwon, Committee Chair

Edwin Sawan, Committee Member

Xiaomi Hu, Committee Member 


\section{DEDICATION}

To my family members and all my teachers 


\section{ACKNOWLEDGEMENTS}

My master's degree would have been difficult to achieve without help from numerous people. I am indebted to them for their advice, guidance, and support throughout my graduate studies.

First, I would like to express my appreciation to my advisor, Dr. Kwon, for his valuable assistance, support, motivation, and patience during the course of my master's degree research. I was always stimulated by his enthusiasm. And I would like to thank Kanghee Lee, my senior officer. I will not forget his countless contributions to me over the past two years. His enthusiasm towards work and insights into research have made him a creative role model for me. Also, I would like to show my sincere thanks to my colleagues in the Wireless Research and Development Group (WiRed group): Sangku, Shuang, Lun, Wenhao, Kenny, Youvaraj, Jessie, Prashanth, Raju, Mahesh, and others. Additionally, I thank Dr. M. Edwin Sawan and Dr. Xiaomi $\mathrm{Hu}$ for being part of the adjudicating committee. Lastly, I express my sincere gratitude to the Republic of Korea Air Force, which provided me the opportunity to study abroad. This experience has proven to be very valuable, and I promise to make a commitment to my country. 


\begin{abstract}
A cooperative amplify-and-forward (AF) wireless relay scheme consisting of $M$ sources, $N$ relays, and $L$ destinations all equipped with a single antenna was studied in this thesis. The main objective was to design jointly and iteratively the closed form of the minimum mean square error (MMSE)-based source precode and relay amplifying matrices under a jamming environment with various power constraints: (1) transmit, (2) aggregate, (3) source, and (4) relay. With the derived optimal source precode and relay amplifying matrices, the jamming influence of various power constraints on system performance were examined numerically using Monte-Carlo simulations.
\end{abstract}




\section{TABLE OF CONTENTS}

\section{Chapter}

Page

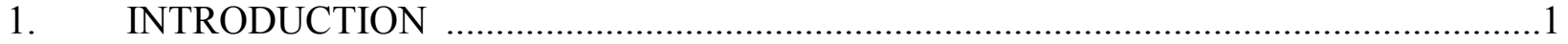

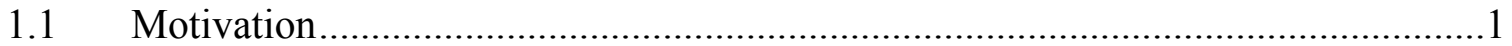

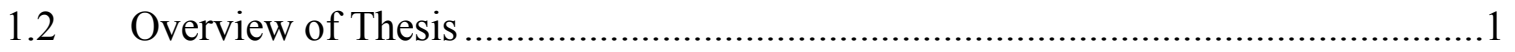

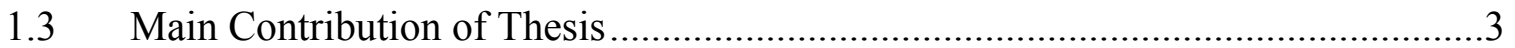

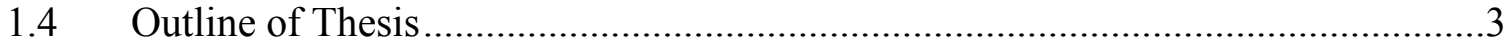

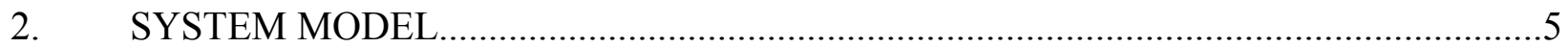

3. COPPERATIVE MMSE WIRELESS RELAY SCHEMES ........................................

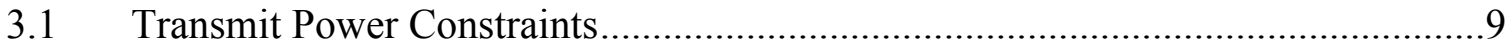

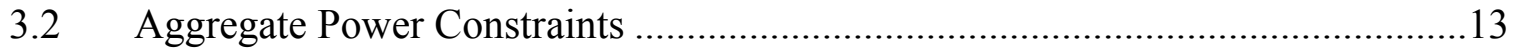

S.3 Source Power Constraints .............................................................................. 14

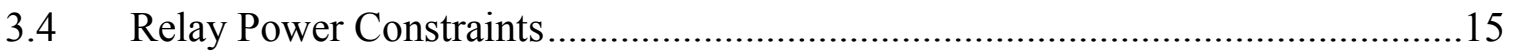

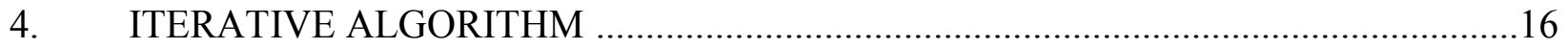

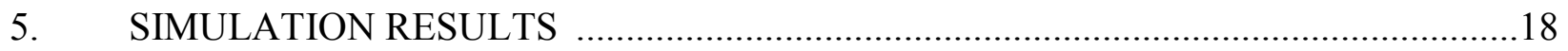

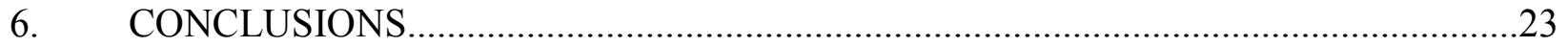

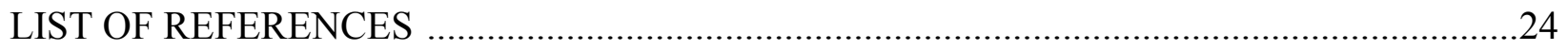

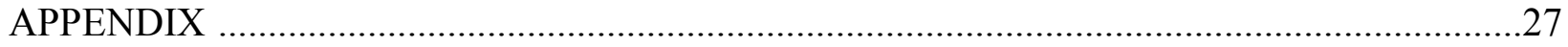




\section{LIST OF FIGURES}

Figure

Page

1. Cooperative AF wireless relay network for $M$ sources, $N$ relays, and, $L$ destinations under jamming environment with various power constraints.

2. BER versus input SNR in AF $M N L$ wireless relay network under no-jamming environment with both transmit power constraint (TPC) and aggregate power constraint (APC) using different number of relays $N=4,6,8 ; M=2$; and $L=2$

3. BER versus input SNR in AF $M N L$ wireless relay network under no-jamming environment with both relay power constraint (RPC) and source power constraint (SPC) using different number of relays $N=6,8 ; M=2$; and $L=2$

4. BER versus input SNR in AF $M N L$ wireless relay network under jamming environment and transmit power constraints with two different jamming conditions, i.e., $E_{b} / N_{J}=$ $13 \mathrm{~dB}$ and $16 \mathrm{~dB}$, using $N=6, M=2, L=2$, and $\gamma=0.3$

5. BER versus PBNJ jamming fraction $\gamma$ with different $E_{b} / N_{J}$, i.e., $0,5,10,15,20 \mathrm{~dB}$, when $E_{b} / N_{J}=13 \mathrm{~dB}$ for cooperative $\mathrm{AF} M N L$ wireless relay network under transmit power constraints using $N=6, M=2$, and $L=2$. 


\section{LIST OF TABLES}

Table

Page

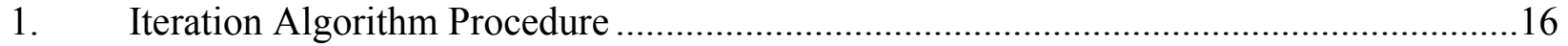




\section{LIST OF ABBREVIATIONS}

$\begin{array}{ll}\text { AF } & \text { Amplify-and-Forward } \\ \text { MIMO } & \text { Multiple-Input Multiple-Output } \\ \text { MNL } & \text { M-source- } N \text {-relay- } L \text {-destination } \\ \text { CSI } & \text { Channel State Information } \\ \text { MMSE } & \text { Minimum Mean Square Error } \\ \text { MSE } & \text { Mean Square Error } \\ \text { PBNJ } & \text { Partial-Band Noise Jamming } \\ \text { BER } & \text { Bit Error Rate } \\ \text { PSD } & \text { Power Spectral Density } \\ \text { AWGN } & \text { Additive White Gaussian Noise } \\ \text { OFDMA } & \text { Orthogonal Frequency Division Multiple Access } \\ \text { TPC } & \text { Transmit Power Constraint } \\ \text { APC } & \text { Aggregate Power Constraint } \\ \text { RPC } & \text { Relay Power Constraint } \\ \text { SPC } & \text { Source Power Constraint } \\ \text { PO } & \end{array}$




\section{CHAPTER 1 \\ INTRODUCTION}

\subsection{Motivation}

It has been assumed in much of the literature that the amplify-and-forward (AF) multipleinput-multiple-output (MIMO) wireless relay system has a one-source-one-relay-one destination system with multiple antennas at each node [1]-[8]. However, the AF multi-source-multi-relaymulti-destination system with a single antenna per node can be more cost effective and practical for the purpose of extending the data range [9]-[13] because all nodes can be arbitrarily located and a node can be a simple sensor under a power constraint. Furthermore, the number of sources cannot be equal to the number of either relays or destinations in a practical system. Hence, this thesis focuses on an AF $M$-source- $N$-relay- $L$-destination $(M N L)$ wireless relay network.

\subsection{Overview of Thesis}

This thesis assumes a cooperative relay network where each relay node and each destination node know the fading channel coefficients. In future wireless generations, e.g., 5G wireless communication systems, this channel state information (CSI) assumption can be feasible because a central service station connected to each relay node station or a small sell base station can provide this CSI or the computed amplifying matrices to each relay node through almost noise free backhauls (i.e., optical fibers). In cooperative wireless relay networks, MIMO relay techniques are well known for achieving the gain of spatial diversity order and the gain of multiplexing during data transmission between sources and destinations, extending the propagation range, and enhancing spectral efficiency [1]-[5]. Hence, the MIMO wireless relay system has gained considerable interest over the past years. Relays in MIMO wireless networks can be either regenerative (also known as decode-and-forward relay) or nonregenerative (also 
known as amplify-and-forward relay). Compared to the former, the latter leads to lower complexity and shorter delay because it only performs the AF function without decoding/encoding operations at the relays. Additionally, the AF relay scheme, which is employed in this thesis, is a reasonable strategy when sources and relays have limited power.

In several research endeavors [5]-[10], the minimum mean square error (MMSE) for a given total transmitted power at the relays was applied to determine a relay amplifying matrix $\mathbf{F}$ for the AF MIMO wireless system using different cost functions. In contrast, the MMSE-based relay amplifying matrix design attempted to minimize the mean square error (MSE) at the destinations for a given total received power at the destinations [11], [12]. In this thesis, the relay amplifying matrix $\mathbf{F}$ is an $N \times N$ matrix in order to minimize the MSE between the received signal (or equalized signal) at the destinations and the originally transmitted signal from the sources, where $N$ is the number of relays. However, a relay amplifying matrix was designed to maximize the capacity for the AF MIMO wireless relay system [4].

In reality, wireless communication nodes are exposed to the risk of either friendly user interference or jamming signals during data transmission. Jamming signals can be intentionally broadcasted by enemies to disrupt friendly user systems [13]. Thus, in this thesis, the AF MIMO wireless relay network was investigated under a broadband noise jamming environment with received power constraints at the destinations [12]. However, partial-band noise jamming (PBNJ) has been reported as a strong jamming strategy when a desired user employs a broadband system [14], [15]. To the best of our knowledge, AF MIMO wireless relay systems under PBNJ have not been analyzed in the literature. Hence, PBNJ is considered for a jamming environment in this thesis. 
Additionally, in this thesis, the transmit power at both the sources and the relays are independently constrained together during data transmission [6], [7]. The sum of the transmit power at the sources and the relays, called aggregate power constraints, is secondly constrained [6], [7]. Finally, the source and the relay power constraints are individually considered [5], [8][10]. In summary, this thesis provides the closed form of the source and relay amplifying matrices for the AF $M N L$ wireless relay network under PBNJ with various power constraints based on MMSE criterion. This thesis has been published, in part, by the author of this thesis with other co-authors [10], [11].

\subsection{Main Contribution of Thesis}

- This is the first thesis treating performance analysis of the one-way distributed AF $M N L$ wireless relay network under a jamming environment.

- The precoder matrices $\mathbf{W}$ at the sources and the relay amplifying matrices $\mathbf{F}$ under both transmit and aggregate power constraints are presented based on the MMSE criterion.

- With the optimal source precoder and relay amplifying matrices derived, the jamming influence on system performance is examined numerically by using Monte-Carlo simulations.

- This thesis presents, for the first time, the worst-jamming fraction numerically for the $\mathrm{AF}$ wireless relay networks, for a given power of jamming signals.

\subsection{Outline of Thesis}

This thesis is organized into five chapters. Chapter 2 describes the system model and data transmission strategies applied. Chapter 3 provides cooperative MMSE relay schemes for AF $M N L$ wireless communication systems under a jamming environment with various power 
constraints. Chapter 4 presents the iterative algorithm. Chapter 5 shows the simulation bit error rate (BER) results. Finally, Chapter 6 concludes the thesis.

Notation: Matrices and vectors are denoted, respectively, by uppercase and lowercase boldface characters (e.g., A and a). The transpose, complex conjugate, inverse, trace, pseudoinverse, and Hermitian of A are denoted, respectively, by $\mathbf{A}^{T}, \mathbf{A}^{*}, \mathbf{A}^{-1}, \operatorname{tr}(\mathbf{A}), \mathbf{A}^{+}$and $\mathbf{A}^{H}$. An $N \times N$ identity matrix is denoted by $\boldsymbol{I}_{N}$. Notations $|a|,\|\mathbf{a}\|$, and $\|\mathbf{A}\|_{F}$ denote the absolute value of $a$ for any scalar, 2-norm of a, and Frobenius-norm of A, respectively. The expectation operator and the real operator are denoted by $E[\cdot]$ and $\operatorname{Re}\{\mathbf{A}\}=\left(\mathbf{A}+\mathbf{A}^{*}\right) / 2$, respectively. 


\section{CHAPTER 2}

\section{SYSTEM MODEL AND DATA TRANSMISSION}

Data transmission between $M$ sources and $L$ destinations through cooperative AF $N$ relays under a jamming environment with various power constraints is shown in Figure 1, where $N \geq$ $M, N \geq L, L \geq M$, and $M \geq L$. Here, it is assumed that all nodes have a single antenna. As shown, there are two stages: where sources broadcast the signal vector $\mathbf{s} \in \mathbf{C}^{M \times 1}=\left[s_{1}, \cdots, s_{M}\right]^{T}$ multiplied by a precode matrix $\mathrm{W}$ under a jamming environment, which is a PBNJ environment (Stage I), and where all relays retransmit their jammed signals from the sources to the destinations multiplied by a relay amplifying matrix $\mathbf{F}$ under another independent PBNJ environment (Stage II). Thus, the destinations receive other independently jammed signals from the relays.

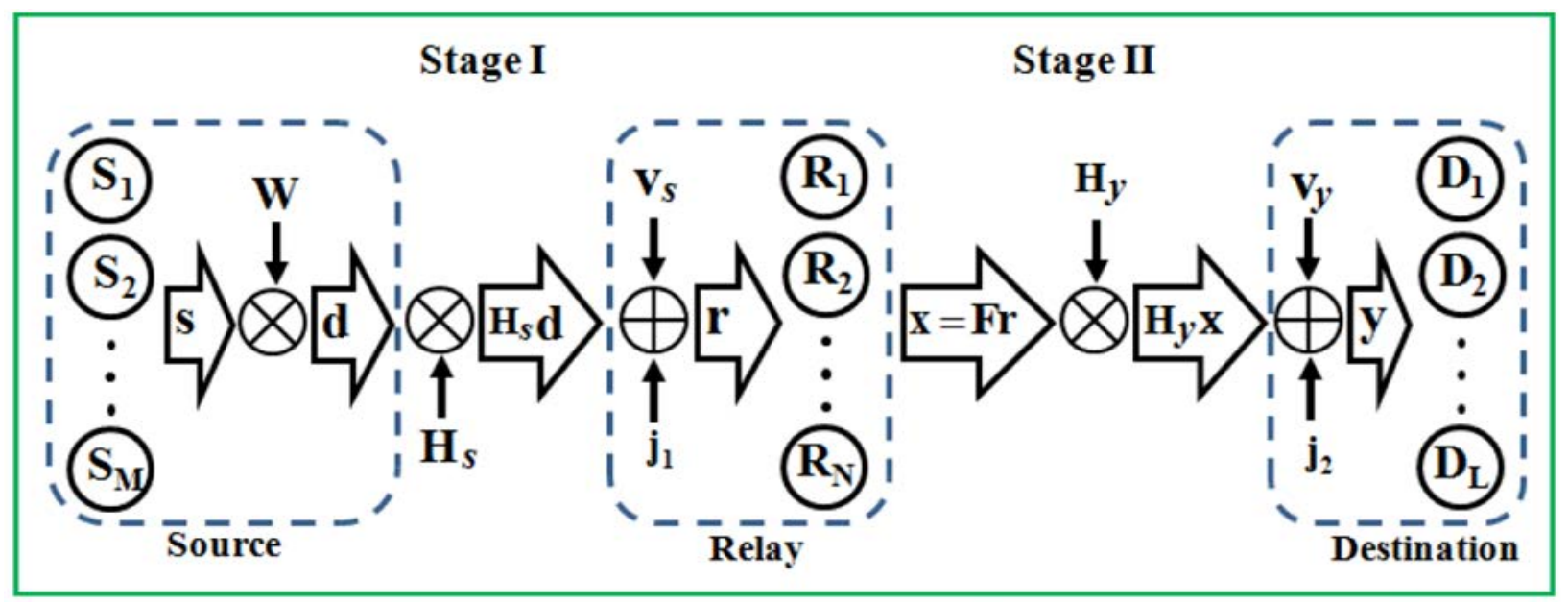

Figure 1. Cooperative AF wireless relay network for $M$ sources, $N$ relays, and, $L$ destinations under jamming environment with various power constraints.

The bandpass noise at a receiver is modeled as a Gaussian noise with the power spectral density (PSD) in a rectangular shape, e.g., the PSD of a bandpass additive white Gaussian noise (AWGN) with height $\frac{N_{O}}{2}$ over the band $\left|f-f_{c}\right| \leq \frac{B W}{2}$, else zero. Similarly, the PBNJ can be 
modeled as a bandpass Gaussian with $\operatorname{PSD} \frac{N_{J}}{2 \gamma}$ over $\left|f-f_{O}\right| \leq \frac{\gamma B W}{2}$, else zero. Here, $f_{c}$ and $f_{O}$ denote the carrier frequency of the signal and the center frequency of the PBNJ, respectively, $\gamma$ is the PBNJ jamming fraction, $0<\gamma<1$, and $B W$ is the system bandwidth. Hence, the PBNJ is modeled as a complex Gaussian noise with zero mean and power $\frac{N_{J} B W}{\gamma}$. In this thesis, the $B W$ is normalized to 1 . And $N_{J} \triangleq \frac{J}{B W}$ denotes the one-sided PSD of a jammer with total jamming power $J$, and the PBNJ fraction $\gamma$ is also equal to the probability of a jamming signal presence during the desired signal transmission in a frequency-hopped system. For example, in an orthogonal frequency division multiple access (OFDMA), a user subcarrier can be jammed with probability because the subcarrier can be located in the $\gamma B W$ band out of the total $B W$. Here, the PBNJ column vectors $\mathbf{j}_{1} \in \mathbf{C}^{N \times 1}$ and $\mathbf{j}_{2} \in \mathbf{C}^{L \times 1}$ are zero-mean complex AWGN with covariance matrices $\frac{N_{J_{1}}}{\gamma} \mathbf{I}_{N}$ and $\frac{N_{J_{2}}}{\gamma} \mathbf{I}_{L}$, respectively.

Let $\mathbf{H}_{s} \in \mathbf{C}^{N \times M}$ denote the complex channel matrix from sources to relays as

$$
\mathbf{H}_{s}=\left[\mathbf{h}_{s, 1}, \mathbf{h}_{s, 2}, \cdots, \mathbf{h}_{s, M}\right]_{-}^{-}
$$

where $\mathbf{h}_{s, m} \in \mathbf{C}^{N \times 1}=\left[h_{s, m, 1}, \cdots, h_{s, m, N}\right]^{T}, m=1, \cdots, M$, is a column vector, representing the channel coefficient from the $m$-th source to all relays. It is assumed that each channel $h_{s, m, N}$ is independent identically distributed (i.i.d.) with a zero-mean and unit-variance circular complex Gaussian and quasi-static Rayleigh fading. Therefore, the received signal complex column vector $\mathbf{r} \in \mathbf{C}^{N \times 1}$ at the relays can be written as

$$
\mathbf{r}=\mathbf{H}_{s} \mathbf{d}+\mathbf{v}_{s}+\mathbf{j}_{1}
$$

where $\mathbf{v}_{s} \in \mathbf{C}^{N \times 1}$ is a zero-mean complex thermal AWGN vector with covariance matrix $\sigma_{\mathrm{v}_{s}} \mathbf{I}_{N}$ and $\mathbf{d} \in \mathbf{C}^{M \times 1} \triangleq \mathbf{W s}$ is a transmitted signal vector from the sources after precoding. Here, 
$\mathbf{W} \in \mathbf{C}^{M \times M}$ denotes a precode matrix at the source. In this thesis, the propagation path loss is not considered. However, it can be included in $\mathbf{W}$ with not much difficulty.

Let $\mathbf{H}_{y} \in \mathbf{C}^{L \times N}$ denote the complex channel matrix from relays to destinations as

$$
\mathbf{H}_{y}=\left[\mathbf{h}_{y, 1}, \mathbf{h}_{y, 2}, \cdots, \mathbf{h}_{y, L}\right]^{T}
$$

where $\mathbf{h}_{y, m}=\left[h_{y, l, 1}, \cdots, h_{y, l, N}\right], l=1, \cdots, L$, is a row vector, representing the channel coefficient from all relay nodes to the $l$-th destination. It is also assumed that each channel coefficient $\mathbf{h}_{y, l, N}$ is i.i.d. with a zero-mean and unit variance circular complex Gaussian and quasi-static Rayleigh fading.

The amplified signal complex column vector $\mathbf{x} \in \mathbf{C}^{N \times 1}$ at the relay outputs is given by

$$
\mathbf{x}=\mathbf{F r}
$$

where $\mathbf{F} \in \mathbf{C}^{N \times N}$ is a relay amplifying matrix employed by the relays to minimize the MSE between the equalized signals at the destinations and the originally transmitted signals from the sources. The received complex signal column vector $\mathbf{y} \in \mathbf{C}^{L \times 1}$ at the destinations can be written as

$$
\mathbf{y}=\mathbf{H}_{y} \mathbf{x}+\mathbf{j}_{2}+\mathbf{v}_{y}
$$

where $\mathbf{v}_{y} \in \mathbf{C}^{L \times 1}$ is a zero-mean complex thermal AWGN vector with covariance matrix ${ }_{\mathbf{v}_{\mathbf{y}}}^{2} \mathbf{I}_{L}$. By substituting equations (2) and (4) into equation (5), $\mathbf{y} \in \mathbf{C}^{L \times 1}$ in equation (5) can be rewritten as

$$
\mathbf{y}=\mathbf{H}_{y} \mathbf{F} \mathbf{H}_{s} \mathbf{W} \mathbf{s}+\mathbf{H}_{y} \mathbf{F} \mathbf{v}_{s}+\mathbf{H}_{y} \mathbf{F} \mathbf{j}_{1}+\mathbf{j}_{2}+\mathbf{v}_{y}
$$

Note that $P B N J \mathbf{j}_{1}$ and $P B N J \mathbf{j}_{2}$ are independent in practice, and $\operatorname{Pr}\left(\right.$ signal jammed by $\left.\mathbf{j}_{1}\right)=\gamma_{1}$ and $\operatorname{Pr}\left(\right.$ signal jammed by $\left.\mathbf{j}_{2}\right)=\gamma_{2}$. For simplicity, it is assumed that $\gamma_{1}=\gamma_{2}=\gamma$. Hence, the average probability of symbol (bit) error $\operatorname{Pr}(e)$ can be written as

$$
P_{r}(e)=\gamma P_{r}(\text { eljammed })+(1-\gamma) P_{r}(\text { elunjammed })
$$


assuming that transmitted signals occupy a narrowband randomly in a broadband system, like an OFDM. This is because $\gamma$ represents both the PBNJ jamming fraction and the probability of a user band being jammed in the broadband system. In the next section, the closed forms of $\mathbf{W}$ and F will be determined by using the MMSE criterion. It is assumed that the channel coefficients are estimated using pilot symbols while the jamming signal is absent, or the central service station directly provides the optimal relay amplifying matrix $\mathbf{F}$ and precoding matrix $\mathbf{W}$ to the relay nodes with the available CSI through the backhauls. 


\section{CHAPTER 3}

\section{COOPERATIVE MMSE WIRELESS RELAY SCHEMES}

In this chapter, the optimum precode matrix $\mathbf{W}$ at the sources and amplifying matrix $\mathbf{F}$ at the relays under PBNJ are derived with various power constraints: (1) transmit, (2) aggregate, (3) source, and (4) relay. Note that although a power constraint at a node is applied, this does not mean a no-power constraint at another node. For example, a relay power constraint does not mean that power can be infinite at the source. Regardless of the power constraints, power at the source and the relay is limited to 1 . Power constraints are used to find the optimal precode matrix $\mathbf{W}$ and relay amplifying matrix $\mathbf{F}$ in the Lagrangian objective function.

\subsection{Transmit Power Constraints}

The main objective in this subsection is to determine optimum $\mathbf{W}^{\dagger}$ and $\mathbf{F}^{\dagger}$ to minimize the MSE between the originally transmitted signal vector from the sources and the received signal vector at the destinations under transmit power constraints at both the sources and the relays. Here, the transmit power of the source and the relay signals are constrained to $P_{S}$ and $P_{R}$, respectively and independently. Therefore, the desired optimization problem under the transmit power constraints at the sources and the relays can be written as

$$
\begin{aligned}
\left(\mathbf{W}^{\dagger}, \mathbf{F}^{\dagger}, \alpha^{\dagger}\right) & =\arg \min _{\mathbf{W}, \mathbf{F}, \alpha} J(\mathbf{W}, \mathbf{F}, \alpha) \\
\text { s.t. } & E\left[\|\mathbf{d}\|^{2}\right]=\mathrm{P}_{\mathrm{S}} \text { and } E\left[\|\mathbf{x}\|^{2}\right]=\mathrm{P}_{\mathrm{R}}
\end{aligned}
$$

where the superscript $\dagger$ means the optimum. The cost function $J(\mathbf{W}, \mathbf{F}, \alpha) \triangleq E\left[\|\mathbf{s}-\widehat{\boldsymbol{y}}\|^{2}\right]$ with $\hat{\mathbf{y}}=\alpha^{-1} \mathbf{y}$ is written as

$$
\begin{aligned}
J(\mathbf{W}, \mathbf{F}, \alpha)= & \alpha^{-2} \sigma_{s}^{2}\left\|\mathbf{H}_{y} \mathbf{F} \mathbf{H}_{s} \mathbf{W}\right\|_{F}^{2}+\alpha^{-2} \beta\left\|\mathbf{H}_{y} \mathbf{F}\right\|_{F}^{2}+M \sigma_{s}^{2} \\
& -2 \alpha^{-1} \sigma_{s}^{2} \operatorname{tr}\left(\operatorname{Re}\left[\mathbf{H}_{y} \mathbf{F} \mathbf{H}_{s} \mathbf{W}\right]\right)+\alpha^{-2} \omega L
\end{aligned}
$$


where $\beta={\sigma_{v_{s}}}^{2}+\frac{N_{J_{1}}}{\gamma}, \quad \omega={\sigma_{v_{y}}}^{2}+\frac{N_{J_{2}}}{\gamma}, E\left[\left|s_{i}\right|^{2}\right]={\sigma_{s_{i}}}^{2},{\sigma_{s_{1}}}^{2}=\cdots={\sigma_{S_{M}}}^{2}={\sigma_{s}}^{2},{\sigma_{v_{s_{1}}}}^{2}=$

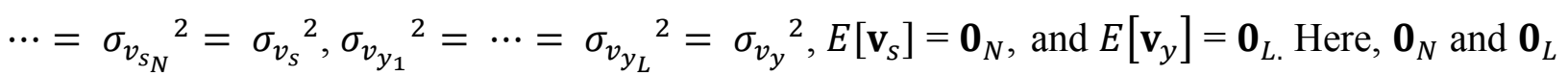
are $N \times 1$ and $L \times 1$ vectors consisting of all zero entries, respectively. And $\hat{\mathbf{y}}$ is defined as $\alpha^{-1} \mathbf{y}$ where the scalar $\alpha$ can be interpreted as a positive gain factor for the MSE in real MNL systems. The total powers $P_{S}$ at the sources and $P_{R}$ at the relays in equation (9) can be written as

$$
\begin{gathered}
\mathbf{P}_{\mathrm{s}}=\sigma_{s}^{2}\|\mathbf{W}\|_{F}^{2} \\
\mathbf{P}_{\mathrm{R}}=\sigma_{s}^{2}\left\|\mathbf{F} \mathbf{H}_{s} \mathbf{W}\right\|_{F}^{2}+\beta\|\mathbf{F}\|_{F}^{2}
\end{gathered}
$$

To determine the constrained optimization problem with the transmit power constraints, the Lagrangian multipliers $\lambda_{S}$ and $\lambda_{R}[16]$ can be applied as

$$
L\left(\mathbf{W}, \mathbf{F}, \alpha, \lambda_{\mathrm{S}}, \lambda_{\mathrm{R}}\right)=J(\mathbf{W}, \mathbf{F}, \alpha)+\lambda_{\mathrm{S}}\left(E\left[\|\mathbf{d}\|^{2}\right]-\mathrm{P}_{\mathrm{S}}\right)+\lambda_{\mathrm{R}}\left(E\left[\|\mathbf{x}\|^{2}\right]-\mathrm{P}_{\mathrm{R}}\right)
$$

For convenience, the constrained Lagrangian optimization $L\left(\mathbf{W}, \mathbf{F}, \alpha, \lambda_{s}, \lambda_{R}\right)$ in equation (13) is henceforth simply stated as $L(\mathbf{F}, \lambda)$. Taking the partial derivative of $L(\mathbf{F}, \lambda)$ with regard to $\mathbf{W}^{*}, \mathbf{F}^{*}, \alpha, \lambda_{S}$ and $\lambda_{R}$, respectively, and using the linear and nonlinear properties of the complex matrix derivative [17] and the cyclic properties of the trace function [18], i.e., $\operatorname{tr}\left(\mathrm{A}_{1} \mathrm{~A}_{2} \mathrm{~A}_{3}\right)=\operatorname{tr}\left(\mathrm{A}_{2} \mathrm{~A}_{3} \mathrm{~A}_{1}\right)$, result in

$$
\begin{aligned}
\frac{\partial L(\mathbf{F}, \lambda)}{\partial \mathbf{F}^{*}}= & \alpha^{-2} \sigma_{s}^{2} \mathbf{H}_{y}^{H} \mathbf{H}_{y} \mathbf{F} \mathbf{H}_{s} \mathbf{W} \mathbf{W}^{H} \mathbf{H}_{s}^{H}+\alpha^{-2} \beta \mathbf{H}_{y}^{H} \mathbf{H}_{y} \mathbf{F} \\
& -\alpha^{-1} \sigma_{s}^{2} \mathbf{H}_{y}^{H} \mathbf{W}^{H} \mathbf{H}_{s}^{H}+\lambda_{\mathrm{R}} \sigma_{s}^{2} \mathbf{F} \mathbf{H}_{s} \mathbf{W} \mathbf{W}^{H} \mathbf{H}_{s}^{H} \\
& +\beta \lambda_{\mathrm{R}} \mathbf{F}=\mathbf{0}_{N} \times_{N} \\
\frac{\partial L(\mathbf{F}, \lambda)}{\partial \mathbf{W}^{*}}= & \alpha^{-2} \sigma_{s}^{2} \mathbf{H}_{s}^{H} \mathbf{F}^{H} \mathbf{H}_{y}^{H} \mathbf{H}_{y} \mathbf{F} \mathbf{H}_{s} \mathbf{W}-\alpha^{-1} \sigma_{s}^{2} \mathbf{H}_{s}^{H} \mathbf{F}^{H} \mathbf{H}_{y}^{H} \\
& +\lambda_{\mathrm{s}} \sigma_{s}^{2} \mathbf{W}+\lambda_{\mathrm{R}} \sigma_{s}^{2} \mathbf{H}_{s}^{H} \mathbf{F}^{H} \mathbf{F} \mathbf{H}_{s} \mathbf{W}=\mathbf{0}_{M \times_{M}}
\end{aligned}
$$




$$
\begin{aligned}
\frac{\partial L(\mathbf{F}, \lambda)}{\partial \alpha}= & 2 \alpha^{-2} \sigma_{s}^{2} \operatorname{tr}\left(\operatorname{Re}\left[\mathbf{H}_{y} \mathbf{F} \mathbf{H}_{s} \mathbf{W}\right]\right)-2 \alpha^{-3} \beta\left\|\mathbf{H}_{y} \mathbf{F}\right\|_{F}^{2} \\
& -2 \alpha^{-3} \sigma_{s}^{2}\left\|\mathbf{H}_{y} \mathbf{F} \mathbf{H}_{s} \mathbf{W}\right\|_{F}^{2}-2 \alpha^{-3} \omega L=0 \\
\frac{\partial L(\mathbf{F}, \lambda)}{\partial \lambda_{\mathrm{s}}}= & \sigma_{s}^{2}\|\mathbf{W}\|_{F}^{2}-\mathrm{P}_{\mathrm{s}}=0 \\
\frac{\partial L(\mathbf{F}, \lambda)}{\partial \lambda_{\mathrm{R}}}= & \sigma_{s}^{2}\left\|\mathbf{F H}_{s} \mathbf{W}\right\|_{F}^{2}+\beta\|\mathbf{F}\|_{F}^{2}-\mathrm{P}_{\mathrm{R}}=0
\end{aligned}
$$

where $\mathbf{0}_{N \times N}$ and $\mathbf{0}_{M \times M}$ are $N \times N$ and $M \times M$ matrices consisting of all zero entries, respectively. Applying algebra tactics to equation (14) results in

$$
\mathbf{F}^{\dagger}=\alpha \sigma_{s}^{2}\left(\mathbf{H}_{u}^{H} \mathbf{H}_{y}+\lambda_{\mathrm{R}} \alpha^{2} \mathbf{I}_{N}\right)^{-1} \mathbf{H}_{a} \mathbf{H}_{b}^{-\mathbf{1}}
$$

where $\mathbf{H} \triangleq \mathbf{H}_{\mathrm{y}}^{\mathrm{H}} \mathbf{W}^{H} \mathbf{H}_{s}^{H}$ and $\mathbf{H}_{\mathrm{b}} \triangleq \sigma_{s}{ }^{2} \mathbf{H}_{\mathrm{s}} \mathbf{W} \mathbf{W}^{H} \mathbf{H}_{s}^{H}+\beta \mathbf{I}_{N}$. As shown in equation (19), it is not easy to determine the Lagrangian multiplier $\lambda_{R}$ due to the first matrix inversion term in equation (19). Hence, to determine the explicit Lagrangian multiplier $\lambda_{R}$, first multiplying $\alpha^{2} \mathbf{F}^{H}$ and then applying the trace function on both sides in equation (14) results in

$$
\alpha \sigma_{s}^{2} \operatorname{tr}\left(\mathbf{H}_{y} \mathbf{F} \mathbf{H}_{s} \mathbf{W}\right)=\sigma_{s}^{2}\left\|\mathbf{H}_{y} \mathbf{F} \mathbf{H}_{s} \mathbf{W}\right\|_{F}^{2}+\beta\left\|\mathbf{H}_{y} \mathbf{F}\right\|_{F}^{2}+\lambda_{\mathrm{R}} \alpha^{2} \mathrm{P}_{\mathrm{R}}
$$

using $\mathbf{P}_{\mathrm{R}}=\sigma_{s}{ }^{2}\left\|\mathbf{F} \mathbf{H}_{\mathbf{s}} \mathbf{W}\right\|_{F}^{2}+\beta\|\mathbf{F}\|_{F}^{2}$. In addition, using $(16), \alpha \sigma_{s}{ }^{2} \operatorname{tr}\left(\mathbf{H}_{\mathrm{y}} \mathbf{F} \mathbf{H}_{s} \mathbf{W}\right)$ can be written as

$$
\alpha \sigma_{s}^{2} \operatorname{tr}\left(\mathbf{H}_{y} \mathbf{F} \mathbf{H}_{s} \mathbf{W}\right)=\sigma_{s}^{2}\left\|\mathbf{H}_{y} \mathbf{F} \mathbf{H}_{s} \mathbf{W}\right\|_{F}^{2}+\beta\left\|\mathbf{H}_{y} \mathbf{F}\right\|_{F}^{2}+L \omega
$$

using the fact that $\operatorname{tr}\left(\operatorname{Re}\left[\mathbf{H}_{y} \mathbf{F} \mathbf{H}_{s} \mathbf{W}\right]\right)=\operatorname{tr}\left(\boldsymbol{H}_{y} \mathbf{F} \mathbf{H}_{s} \mathbf{W}\right)$ when $\operatorname{tr}\left(\mathbf{H}_{y} \mathbf{F} \mathbf{H}_{s} \mathbf{W}\right)=\operatorname{tr}\left(\mathbf{W}^{H} \mathbf{H}_{s}^{H} \mathbf{F}^{H} \mathbf{H}_{y}^{H}\right)$, which can be verified by substituting equation (19) into $\operatorname{tr}\left(\mathbf{H}_{y} \mathbf{F} \mathbf{H}_{s} \mathbf{W}\right)$ and $\operatorname{tr}\left(\mathbf{W}^{H} \mathbf{H}_{s}^{H} \mathbf{F}^{H} \mathbf{H}_{y}^{H}\right)$, respectively. Comparing equations (20) and (21), $\lambda_{R} \alpha^{2}$ can be obtained as

$$
\lambda_{\mathrm{R}} \alpha^{2}=\frac{L \omega}{\mathrm{P}_{\mathrm{R}}}
$$


After first substituting equation (22) into equation (19), and then substituting equation (19) into equation (18), the optimal $\alpha^{\dagger}$ under PBNJ with the transmit power constraints can be written as

$$
\alpha^{\dagger}=\frac{\sigma_{s}^{-2} \sqrt{\mathrm{P}_{\mathrm{R}}}}{\sqrt{\sigma_{s}^{2}\left\|\mathbf{H}_{t}^{-1} \mathbf{H}_{a} \mathbf{H}_{b}^{-1} \mathbf{H}_{s} \mathbf{W}\right\|_{F}^{2}+\beta\left\|\mathbf{H}_{t}^{-1} \mathbf{H}_{a} \mathbf{H}_{b}^{-1}\right\|_{F}^{2}}}
$$

where $\mathbf{H}_{\mathrm{t}}=\mathbf{H}_{y}^{H} \mathbf{H}_{y}+L \omega \mathrm{P}_{\mathrm{R}}^{-1} \mathbf{I}_{N}$. Using equations (22) and (23), the optimal $\lambda_{\mathrm{R}}^{\dagger}$ under PBNJ with the transmit power constraints can be obtained as

$$
\lambda_{\mathrm{R}}^{\dagger}=\frac{\sigma_{s}^{4} L \omega \mathrm{P}_{\mathrm{R}}^{-2}}{\left(\sigma_{s}^{2}\left\|\mathbf{H}_{t}^{-1} \mathbf{H}_{a} \mathbf{H}_{b}^{-1} \mathbf{H}_{s} \mathbf{W}\right\|_{F}^{2}+\beta\left\|\mathbf{H}_{t}^{-1} \mathbf{H}_{a} \mathbf{H}_{b}^{-1}\right\|_{F}^{2}\right)^{-1}}
$$

Finally, the optimal $\mathbf{F}^{\dagger}$ under PBNJ with the transmit power constraints can be written as

$$
\mathbf{F}^{\dagger}=\frac{\mathbf{H}_{t}^{-1} \mathbf{H}_{a} \mathbf{H}_{b}^{-1} \sqrt{\mathrm{P}_{\mathrm{R}}}}{\sqrt{\sigma_{s}^{2}\left\|\mathbf{H}_{t}^{-1} \mathbf{H}_{a} \mathbf{H}_{b}^{-1} \mathbf{H}_{s} \mathbf{W}\right\|_{F}^{2}+\beta\left\|\mathbf{H}_{t}^{-1} \mathbf{H}_{a} \mathbf{H}_{b}^{-1}\right\|_{F}^{2}}}
$$

Similarly, using equations (15) and (17), the optimal $\mathbf{W}^{\dagger}$ and $\lambda_{\mathrm{S}}^{\dagger}$ under PBNJ with the transmit power constraints can be written as

$$
\begin{gathered}
\mathbf{W}^{\dagger}=\frac{\mathbf{H}_{k}^{-1} \mathbf{H}_{s}^{H} \mathbf{F}^{H} \mathbf{H}_{y}^{H} \sqrt{\mathrm{P}_{\mathrm{s}}}}{\sqrt{\sigma_{s}^{2}\left\|\mathbf{H}_{k}^{-1} \mathbf{H}_{s}^{H} \mathbf{F}^{H} \mathbf{H}_{y}^{H}\right\|_{F}^{2}}} \\
\lambda_{\mathrm{s}}^{\dagger}=\varepsilon \sigma_{s}^{2} \mathrm{P}_{\mathrm{s}}^{-1}\left\|\mathbf{H}_{k}^{-1} \mathbf{H}_{s}^{H} \mathbf{F}^{H} \mathbf{H}_{y}^{H}\right\|_{F}^{2}
\end{gathered}
$$

where

$$
\begin{gathered}
\mathbf{H}_{k}=\mathbf{H}_{s}^{H} \mathbf{F}^{H} \mathbf{H}_{y}^{H} \mathbf{H}_{y} \mathbf{F} \mathbf{H}_{s}+L \omega \mathrm{P}_{\mathrm{R}}^{-1} \mathbf{H}_{s}^{H} \mathbf{F}^{H} \mathbf{F} \mathbf{H}_{s}+\varepsilon \mathbf{I}_{M} \\
\varepsilon=\frac{\beta\left\|\mathbf{H}_{\mathrm{y}} \mathbf{F}\right\|_{F}^{2}-\frac{\omega \beta L}{\mathrm{P}_{\mathrm{R}}}\|\mathbf{F}\|_{F}^{2}}{\mathrm{P}_{\mathbf{S}}}
\end{gathered}
$$

Note that the optimal solutions $\left\{\mathbf{W}_{\mathrm{NOJ}}^{\dagger}, \mathbf{F}_{\mathrm{NOJ}}^{\dagger}, \alpha_{\mathrm{NOJ}}^{\dagger}, \lambda_{\mathrm{S}-\mathrm{NOJ}}^{\dagger}, \lambda_{\mathrm{R}-\mathrm{NOJ}}^{\dagger}\right\}$ for the AF $M N L$ wireless relay network under a no-jamming environment with transmit power constraints at the sources 
and the relays can be derived with $\frac{N_{J_{1}}}{\gamma}=\frac{N_{J_{2}}}{\gamma}=0$ in equations (23) to (26) and equation (27). Here, the subscript noJ refers to the case of the no-jamming environment with the transmit power constraints at the sources and the relays.

\subsection{Aggregate Power Constraints}

In this subsection, the sum of transmit power at the sources and the relays, defined as aggregate power constraints in the introduction, is constrained to $P_{T}$, where $P_{T}=P_{S}+P_{R}$. Accordingly, the desired optimization problem under PBNJ with the aggregate power constraints can be modified as

$$
\begin{aligned}
& \left(\mathbf{W}^{\dagger}, \mathbf{F}^{\dagger}, \alpha^{\dagger}\right)=\arg \min _{\mathbf{W}, \mathbf{F}, \alpha} J(\mathbf{W}, \mathbf{F}, \alpha) \\
& \text { s.t. } E\left[\|\mathbf{d}\|^{2}\right]+E\left[\|\mathbf{x}\|^{2}\right]=\mathrm{P}_{\mathrm{T}}
\end{aligned}
$$

Hence, the constrained Lagrangian optimization with the aggregate power constraints can be written as

$$
L\left(\mathbf{W}, \mathbf{F}, \alpha, \lambda_{\mathrm{T}}\right)=J(\mathbf{W}, \mathbf{F}, \alpha)+\lambda_{\mathrm{T}}\left(E\left[\|\mathbf{d}\|^{2}\right]+E\left[\|\mathbf{x}\|^{2}\right]-\mathbf{P}_{\mathrm{T}}\right)
$$

where the aggregate power $\mathbf{P}_{\mathrm{T}}$ is given by

$$
\mathbf{P}_{\mathrm{T}}=\sigma_{s}^{2}\|\mathbf{W}\|_{F}^{2}+\sigma_{s}^{2}\left\|\mathbf{F} \mathbf{H}_{s} \mathbf{W}\right\|_{F}^{2}+\beta\|\mathbf{F}\|_{F}^{2}
$$

Following the procedures of transmit power constraints, the optimal solutions of $L(\mathbf{W}, \mathbf{F}, \alpha$, $\lambda \mathrm{T}$ in equation (32) under PBNJ with the aggregate power constraints can be obtained, respectively, as

$$
\mathbf{F}^{\dagger}=\frac{\mathbf{H}_{g}^{-1} \mathbf{H}_{a} \mathbf{H}_{b}^{-1} \sqrt{\mathrm{P}_{\mathrm{T}}-\sigma_{s}^{2}\|\mathbf{W}\|_{F}^{2}}}{\sqrt{\sigma_{s}^{2}\left\|\mathbf{H}_{g}^{-1} \mathbf{H}_{a} \mathbf{H}_{b}^{-1} \mathbf{H}_{s} \mathbf{W}\right\|_{F}^{2}+\beta\left\|\mathbf{H}_{g}^{-1} \mathbf{H}_{a} \mathbf{H}_{b}^{-1}\right\|_{F}^{2}}}
$$




$$
\begin{aligned}
\mathbf{W}^{\dagger} & =\frac{\mathbf{H}_{z}^{-1} \mathbf{H}_{s}^{H} \mathbf{F}^{H} \mathbf{H}_{y}^{H} \sqrt{\left(\mathrm{P}_{\mathrm{T}}-\beta\|\mathbf{F}\|_{F}^{2}\right) \sigma_{s}^{-2}}}{\sqrt{\left\|\mathbf{H}_{z}^{-1} \mathbf{H}_{s}^{H} \mathbf{F}^{H} \mathbf{H}_{y}^{H}\right\|_{F}^{2}+\left\|\mathbf{F} \mathbf{H}_{s} \mathbf{H}_{z}^{-1} \mathbf{H}_{s}^{H} \mathbf{F}^{H} \mathbf{H}_{y}^{H}\right\|_{F}^{2}}} \\
\alpha^{\dagger} & =\frac{\sigma_{s}^{-2} \sqrt{\mathbf{P}_{\mathrm{T}}-\sigma_{s}^{2}\|\mathbf{W}\|_{F}^{2}}}{\sqrt{\sigma_{s}^{2}\left\|\mathbf{H}_{g}^{-1} \mathbf{H}_{a} \mathbf{H}_{b}^{-1} \mathbf{H}_{s} \mathbf{W}\right\|_{F}^{2}+\beta\left\|\mathbf{H}_{g}^{-1} \mathbf{H}_{a} \mathbf{H}_{b}^{-1}\right\|_{F}^{2}}} \\
\lambda_{\mathrm{T}}^{\dagger} & =\frac{\sigma_{s}^{2}\left\|\mathbf{H}_{g}^{-1} \mathbf{H}_{a} \mathbf{H}_{b}^{-1} \mathbf{H}_{s} \mathbf{W}\right\|_{F}^{2}+\beta\left\|\mathbf{H}_{g}^{-1} \mathbf{H}_{a} \mathbf{H}_{b}^{-1}\right\|_{F}^{2}}{\sigma_{s}^{-4} \omega^{-1} L^{-1}\left(\mathrm{P}_{\mathrm{T}}-\sigma_{s}^{2}\|\mathbf{W}\|_{F}^{2}\right)^{2}}
\end{aligned}
$$

where

$$
\begin{gathered}
\mathbf{H}_{g}=\mathbf{H}_{y}^{H} \mathbf{H}_{y}+L \omega \mathrm{P}_{\mathrm{R}}{ }^{-1} \mathbf{I}_{N} \\
\mathbf{H}_{z}=\mathbf{H}_{s}^{H} \mathbf{F}^{H} \mathbf{H}_{y}^{H} \mathbf{H}_{y} \mathbf{F} \mathbf{H}_{s}+L \omega \mathrm{P}_{\mathrm{R}}^{-1} \mathbf{H}_{s}^{H} \mathbf{F}^{H} \mathbf{F} \mathbf{H}_{s}+L \omega \mathrm{P}_{\mathrm{R}}^{-1} \mathbf{I}_{M}
\end{gathered}
$$

\subsection{Source Power Constraints}

The power can be constrained only at the sources during data transmission. Hence, as in the case of the two previous power constraints, the desired constrained Lagrangian optimization problem under PBNJ with the source power constraints can be written as

$$
L\left(\mathbf{W}, \mathbf{F}, \alpha, \lambda_{\mathrm{s}}\right)=J(\mathbf{W}, \mathbf{F}, \alpha)+\lambda_{\mathrm{s}}\left(E\left|\|\mathbf{d}\|^{2}\right|-\mathbf{P}_{\mathrm{s}}\right)
$$

Therefore, the solutions of $L\left(\mathbf{W}, \mathbf{F}, \alpha, \lambda_{\mathrm{S}}\right)$ in equation (40) can be obtained, respectively, as

$$
\begin{gathered}
\mathbf{F}^{\dagger}=\alpha \sigma_{s}^{2} \mathbf{H}_{y}^{+} \mathbf{W}^{H} \mathbf{H}_{s}^{H} \mathbf{H}_{b}^{-1} \\
\mathbf{W}^{\dagger}=\frac{\mathbf{H}_{q}^{-1} \mathbf{H}_{s}^{H} \mathbf{F}^{H} \mathbf{H}_{y}^{H} \sqrt{\mathbf{P}_{s}}}{\sqrt{\sigma_{s}^{2}\left\|\mathbf{H}_{q}^{-1} \mathbf{H}_{s}^{H} \mathbf{F}^{H} \mathbf{H}_{y}^{H}\right\|_{F}^{2}}} \\
\alpha^{\dagger}=\frac{\sqrt{\mathbf{P}_{\mathrm{s}}}}{\sqrt{\sigma_{s}^{2}\left\|\mathbf{H}_{q}^{-1} \mathbf{H}_{s}^{H} \mathbf{F}^{H} \mathbf{H}_{y}^{H}\right\|_{F}^{2}}} \\
\lambda_{\mathrm{s}}^{\dagger}=\sigma_{s}^{2}\left\|\mathbf{H}_{q}^{-1} \mathbf{H}_{s}^{H} \mathbf{F}^{H} \mathbf{H}_{y}^{H}\right\|_{F}^{2}\left(\beta\left\|\mathbf{H}_{y} \mathbf{F}\right\|_{F}^{2}+\omega L\right) \mathrm{P}_{\mathrm{s}}^{-2}
\end{gathered}
$$


where

$$
\mathbf{H}_{q}=\mathbf{H}_{s}^{H} \mathbf{F}^{H} \mathbf{H}_{y}^{H} \mathbf{H}_{y} \mathbf{F} \mathbf{H}_{s}+L \omega \mathrm{P}_{\mathrm{T}}^{-1} \mathbf{I}_{M}
$$

Here $\left(\mathbf{H}_{y}^{H} \mathbf{H}_{y}\right)^{+} \mathbf{H}_{y}^{H}=\mathbf{H}_{y}^{+}$is used.

\subsection{Relay Power Constraints}

Using equation (4), only the relay power constraints are constrained during data transmission. Therefore, the MMSE formulation $L\left(\mathbf{W}, \mathbf{F}, \alpha, \lambda_{\mathrm{R}}\right)$ can be written as

$$
L\left(\mathbf{W}, \mathbf{F}, \alpha, \lambda_{\mathrm{R}}\right)=J(\mathbf{W}, \mathbf{F}, \alpha)+\lambda_{\mathrm{R}}\left(E\left[\|\mathbf{x}\|^{2}\right]-\mathrm{P}_{\mathrm{R}}\right)
$$

Therefore, the solutions of $L\left(\mathbf{W}, \mathbf{F}, \alpha, \lambda_{\mathrm{R}}\right)$ in equation (46) can be obtained, respectively, as

$$
\begin{gathered}
\mathbf{F}^{\dagger}=\frac{\mathbf{H}_{t}^{-1} \mathbf{H}_{a} \mathbf{H}_{b}^{-1} \sqrt{\mathrm{P}_{\mathrm{R}}}}{\sqrt{\sigma_{s}^{2}\left\|\mathbf{H}_{t}^{-1} \mathbf{H}_{a} \mathbf{H}_{b}^{-1} \mathbf{H}_{s} \mathbf{W}\right\|_{F}^{2}+\beta\left\|\mathbf{H}_{t}^{-1} \mathbf{H}_{a} \mathbf{H}_{b}^{-1}\right\|_{F}^{2}}} \\
\mathbf{W}^{\dagger}=\frac{\mathbf{H}_{o}^{-1} \mathbf{H}_{s}^{H} \mathbf{F}^{H} \mathbf{H}_{y}^{H} \sqrt{\mathrm{P}_{\mathrm{R}}-\beta\|\mathbf{F}\|_{F}^{2}}}{\sqrt{\sigma_{s}^{2}\left\|\mathbf{F H}_{s} \mathbf{H}_{o}^{-1} \mathbf{H}_{s}^{H} \mathbf{F}^{H} \mathbf{H}_{y}^{H}\right\|_{F}^{2}}} \\
\alpha^{\dagger}=\frac{\sqrt{\mathbf{P}_{\mathrm{R}}-\beta\|\mathbf{F}\|_{F}^{2}}}{\sqrt{\sigma_{s}^{2}\left\|\mathbf{F H}_{s} \mathbf{H}_{o}^{-1} \mathbf{H}_{s}^{H} \mathbf{F}^{H} \mathbf{H}_{y}^{H}\right\|_{F}^{2}}} \\
\lambda_{\mathrm{R}}^{\dagger}=\frac{\sigma_{s}^{4} L \omega \mathrm{P}_{\mathrm{R}}^{-2}}{\left(\sigma_{s}^{2}\left\|\mathbf{H}_{t}^{-1} \mathbf{H}_{a} \mathbf{H}_{b}^{-1} \mathbf{H}_{s} \mathbf{W}\right\|_{F}^{2}+\beta\left\|\mathbf{H}_{t}^{-1} \mathbf{H}_{a} \mathbf{H}_{b}^{-1}\right\|_{F}^{2}\right)^{-1}}
\end{gathered}
$$

where

$$
\mathbf{H}_{o}=\mathbf{H}_{s}^{H} \mathbf{F}^{H} \mathbf{H}_{y}^{H} \mathbf{H}_{y} \mathbf{F} \mathbf{H}_{s}+L \omega \mathrm{P}_{\mathrm{R}}^{-1} \mathbf{H}_{s}^{H} \mathbf{F}^{H} \mathbf{F} \mathbf{H}_{s}
$$




\section{CHAPTER 4}

\section{ITERATIVE ALGORITHM}

Since the optimal $\mathbf{W}^{\dagger}, \mathbf{F}^{\dagger}, \alpha^{\dagger}, \lambda^{\dagger}$ and $\lambda^{\dagger}$ for the cooperative AF $M N L$ wireless relay network under PBNJ with the transmit power constraints at the relays and the sources are functions of one another, they can be solved by an iterative algorithm where variables are calculated one at a time while fixing all others. The proposed iterative algorithm for the cooperative AF $M N L$ wireless relay network under PBNJ with the power constraints at the relays and the sources is stated in Table 1.

TABLE 1

\section{ITERATIVE ALGORITHM UNDER PBNJ WITH TPC}

\begin{tabular}{l|l}
\hline Step 1 & Initialization, $k=0$ \\
& $\mathbf{F}=\mathbf{I}_{N}, L(\mathbf{F}, \lambda)=10$ \\
\hline & Iteration: $k \leftarrow k+1$ \\
& $\mathbf{F}_{k}=f_{\mathbf{F}_{k-1}}\left(\boldsymbol{W}_{k-1}\right)$ \\
& $\mathbf{W}_{k}=f_{\mathbf{W}_{k}}\left(\mathbf{F}_{k}\right)$ \\
& $\alpha=f_{a_{k}}\left(\mathbf{W}_{k}\right)$ \\
& $\lambda_{S}=f_{\lambda_{S_{k}}}\left(\mathbf{F}_{k}\right)$ \\
& $\lambda_{R}=f_{\lambda_{R_{k}}}\left(\mathbf{W}_{k}\right)$ \\
& $\mathrm{L}\left(\mathbf{F}_{k}, \lambda\right)=f_{\mathrm{L}\left(\mathbf{F}_{k}, \lambda\right)}\left(\mathbf{W}_{k}, \mathbf{F}_{k}, \alpha, \lambda_{S}, \lambda_{R}\right)$ \\
& $※$ Applying normalization with respect to $\mathbf{W}_{k}$ and $\mathbf{F}_{k}$ \\
\hline Step 2 & If $0 \leq L\left(\mathbf{F}_{k-1}, \lambda\right)-L\left(\mathbf{F}_{k}, \lambda\right) \leq \eta$ go to step 4 and stop, otherwise go \\
& back to Step 2. $※ \eta=0.0001$ \\
\hline Step 3 4 & $\mathbf{W}=\mathbf{W}_{k} ; \mathbf{F}=\mathbf{F}_{k}$ \\
\hline
\end{tabular}

The constrained Lagrangian optimization $L(\mathbf{F}, \lambda)$ value in each iterative step is monotonically decreased as $k$ increases one by one in Table 1 because the cost function in 
equation (10) is defined as the MMSE [19]. Since the constrained Lagrangian optimization $L(\mathbf{F}, \lambda)$ has positive and convergent properties at all times for one variable with other fixed variables, the difference between $L\left(\mathbf{F}_{k-1}, \lambda\right)$ and $L\left(\mathbf{F}_{k}, \lambda\right)$ can be used as a criterion to stop the iterative algorithm with a designed positive scalar $\eta$ in Step 3.

In addition, as in the case of transmit power constraints at the relays and the source under a jamming environment, using the iterative algorithm, all optimal values for aggregate power constraints and for power constraints only at the sources and only at the relays can be solved, as discussed in Chapter 3. 


\section{CHAPTER 5}

\section{SIMULATION RESULTS}

To evaluate the proposed cooperative AF MNL relay strategy under PBNJ, Monte-Carlo simulations with the following power constraints are performed: (1) transmit power constraints, (2) aggregate power constraints, (3) source power constraints, and (4) relay power constraints. The complex channel matrices $\mathbf{H}_{s}$ and $\mathbf{H}_{y}$ are generated from zero mean and unit variance independent Gaussian random variables. The originally transmitted signals at the source are assumed to be modulated using quadrature phase-shift keying with unit power. Since the MMSE relay scheme is applied in this thesis, the BER is a good criterion to evaluate the proposed system performance. In addition, the total power constraints are set to $\mathrm{P}_{\mathrm{S}}=\mathrm{P}_{\mathrm{R}}=1$ and $\mathrm{P}_{\mathrm{T}}=2$, respectively. As assumed in Chapter 1, all nodes have the same thermal noise power, i.e., $\sigma_{v_{s}}{ }^{2}=$ $\sigma_{v_{y}}{ }^{2}$. Additionally, it is assumed that the number of sources is the same as the number of destinations, i.e., $M=L$. However, this can be simulated for the $M \neq L$ case.

Figure 2 shows the BER versus input SNR in the AF $M N L$ wireless relay network under a no-jamming environment with both transmit power constraints and aggregate power constraints using a different number of relays $N=4,6,8 ; M=2$; and $L=2$, respectively. It is found that the better BER performance is observed as the number of relays increases in both the transmit and aggregate power constraint cases for a given $M$ and $L$. This is because the cost function value decreases as $N$ increases. It is also observed that when the transmit power is constrained, i.e., $E\left[\|\mathbf{d}\|^{2}\right]=\mathrm{P}_{\mathrm{S}}$ and $E\left[\|\mathbf{x}\|^{2}\right]=\mathrm{P}_{\mathrm{R}}$, the BER performance shows a slightly better performance than the one when the aggregate power is constrained, i.e., $E\left[\|\mathbf{d}\|^{2}\right]+E\left[\|\mathbf{x}\|^{2}\right]=\mathrm{P}_{\mathrm{T}}=2$. This is also because the cost function value of the transmit power constraint is slightly smaller than that of the aggregate power constraint. Finally, it can be seen that BER performance of the 
transmit power constraint case is the best among all other power constraint cases shown in Figures 2 and 3. Hence, a designer can apply transmit power constraints at both the sources and the relays for improving system performance of cooperative AF $M N L$ wireless relay networks. If non-optimum $\mathbf{W}$ and $\mathbf{F}$ are used, then the system performances are poor and not comparable.

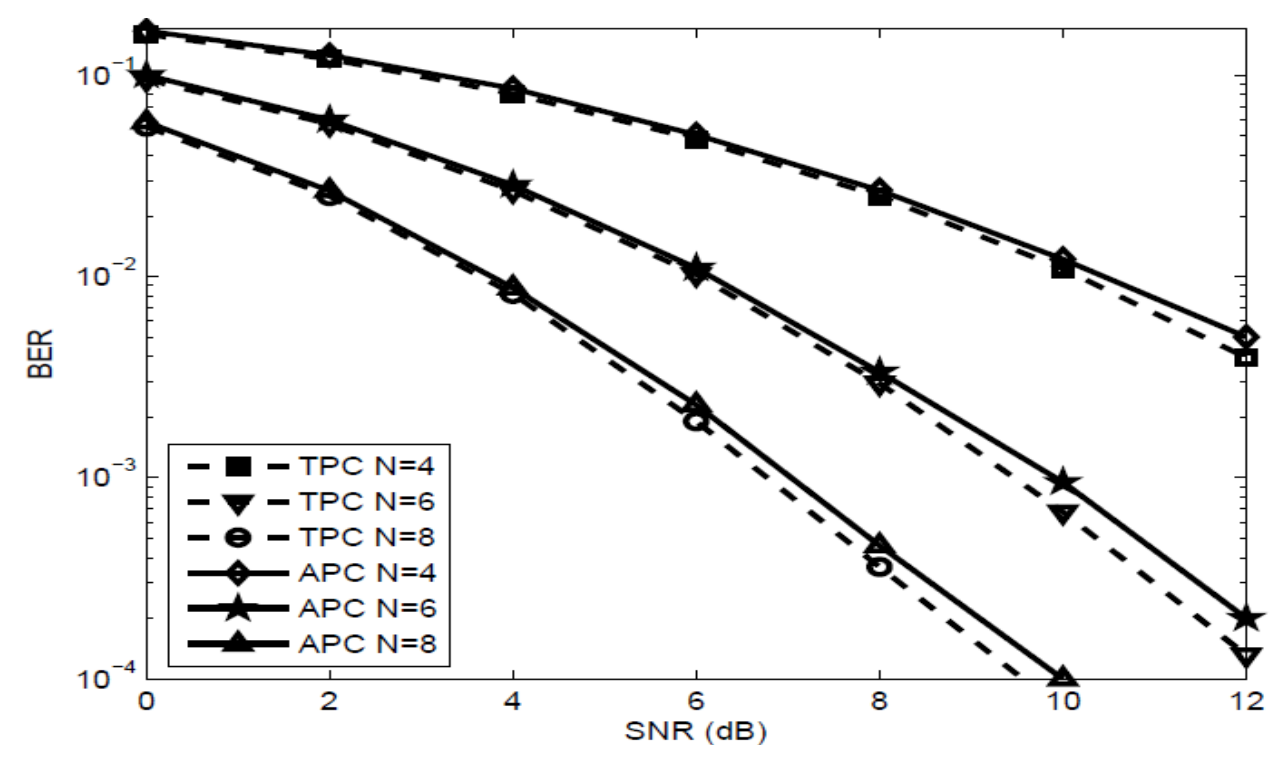

Figure 2. BER versus input SNR in AF $M N L$ wireless relay network under no-jamming environment with both transmit power constraint (TPC) and aggregate power constraint (APC) using different number of relays $N=4,6,8 ; M=2$; and $L=2$, respectively.

Figure 3 presents BER performance versus input SNR in the AF $M N L$ wireless relay network under a no-jamming environment with both relay power constraints and source power constraints using a different number of relays $N=6,8 ; M=2$; and $L=2$, respectively. It is observed that the BER performance under the relay power constraint is better than the one under the source power constraint. For example, the $N=8$ case with the relay power constraint for the cooperative $M N L$ wireless relay network AF shows approximately $1.5 \mathrm{~dB}$ better than the $N=8$ case with the the source power constraint. Hence, when the source precode and relay amplifying matrices are applied, the relay power constraints are more suitable for improving system performance than the source power constraint. 


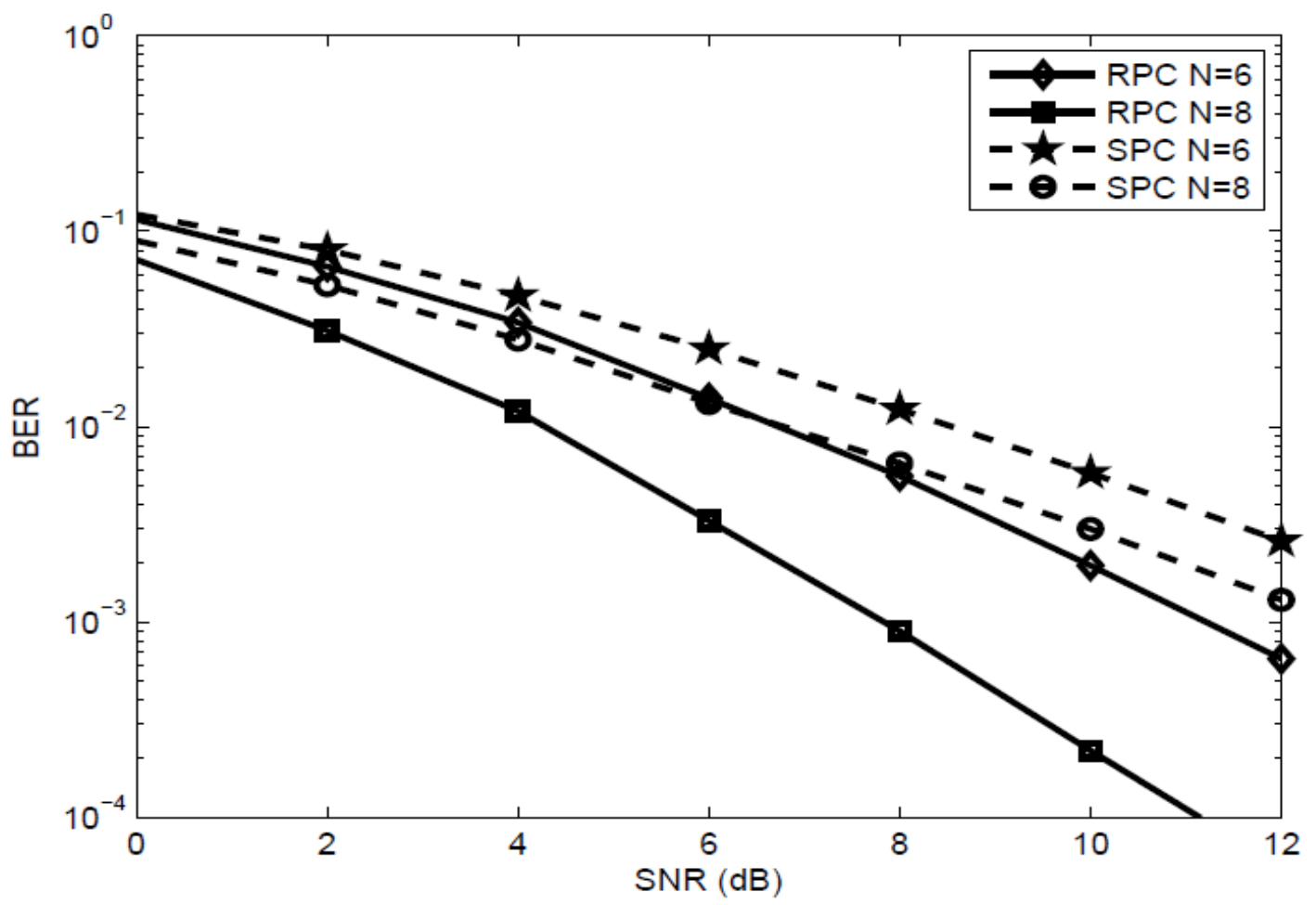

Figure 3. BER versus input SNR in AF $M N L$ wireless relay network under no-jamming environment with both relay power constraint (RPC) and source power constraint (SPC) using different number of relays $N=6,8 ; M=2$, and $L=2$, respectively.

Two different PBNJs, i.e., 2.5\% and 5\% of the desired signal bit energy with $\gamma=0.3$, are modeled as AWGN, respectively. Namely, the variances of the jamming signals are chosen, respectively, to satisfy $10 \log _{10}\left(\frac{\sigma_{s}^{2}}{\sigma_{j_{t}}^{2}}\right)=16 \mathrm{~dB}$ and $13 \mathrm{~dB}$, where $\sigma_{s}^{2}=E_{b}, \sigma_{j_{t}}^{2}=\frac{N_{J_{t}}}{\gamma}, t=1,2$. It is assumed that $\frac{N_{J_{1}}}{\gamma}=\frac{N_{J_{2}}}{\gamma}$. Figure 4 shows BER performance versus input SNR in the cooperative AF $M N L$ wireless relay network under the PBNJ and transmit power constraints with two different jamming conditions, i.e., $13 \mathrm{~dB}$ and $16 \mathrm{~dB}$, using $N=6, M=2, L=2$, and $\gamma=0.3$, respectively. Also, the no-jamming case is presented to compare the difference between the nojamming and jamming environments. As expected, it can be seen that BER performance worsens as the variances of jamming signals increase because the MMSE cost function values under PBNJ increase. As can be seen, the relay power constraint less negatively affects system 
performance compared to the aggregate power constraint, due to the effect of the optimal source precode and relay amplifying matrices.

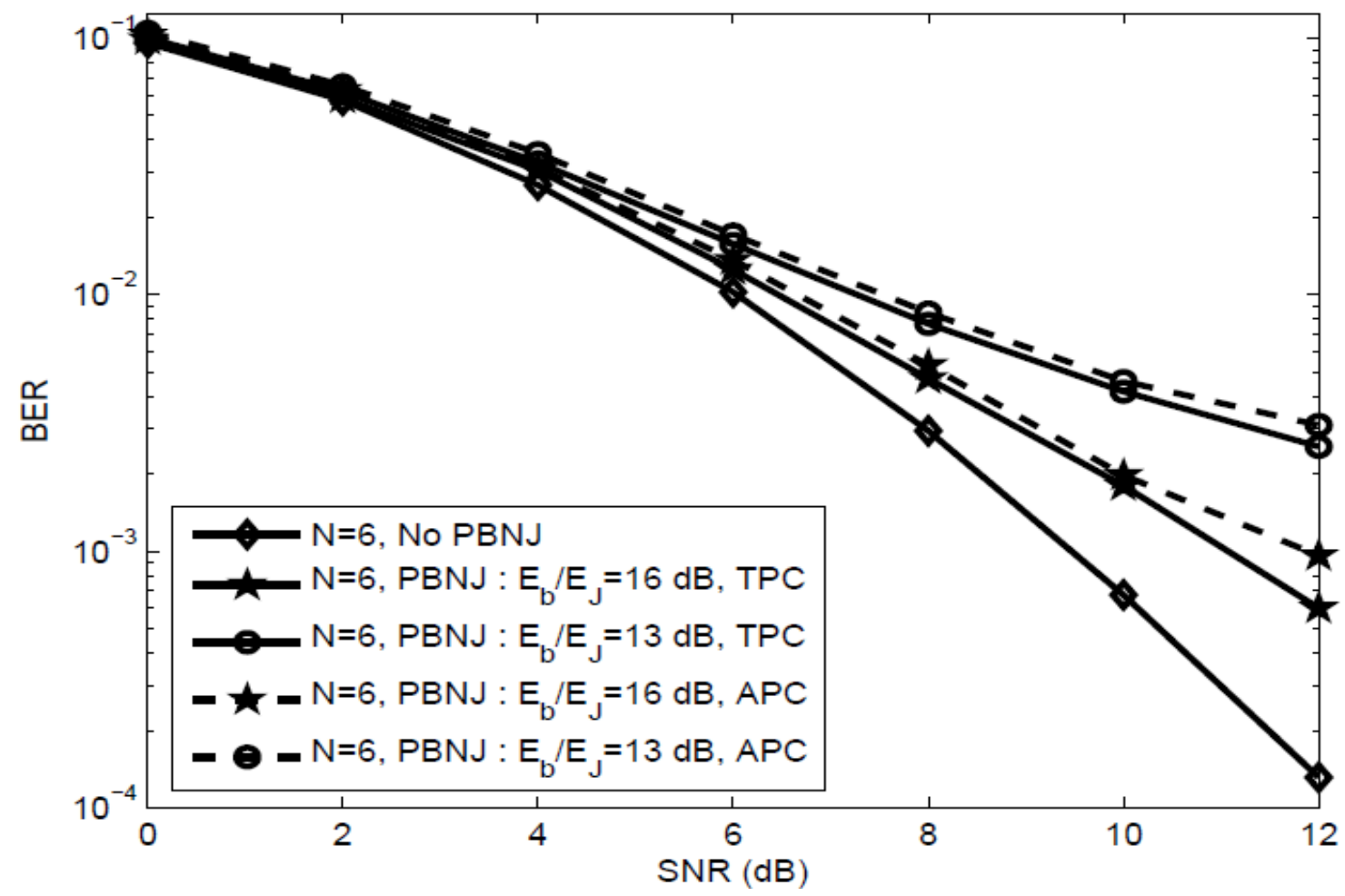

Figure 4. BER versus input $\mathrm{SNR}$ in $\mathrm{AF} M N L$ wireless relay network under jamming environment and transmit power constraints with two different jamming conditions, i.e., $E_{b} / N_{J}$ $=13 \mathrm{~dB}$ and $16 \mathrm{~dB}$, using $N=6, M=2, L=2$, and $\gamma=0.3$, respectively.

Figure 5 shows the BER versus the PBNJ jamming fraction $\gamma$ with different $E_{b} / N_{J}$, i.e., $0,5,10,15,20 \mathrm{~dB}$, when $E_{b} / N_{J}=13 \mathrm{~dB}$ for the cooperative AF $M N L$ wireless relay network under transmit power constraints using $N=6, M=2$, and $L=2$, respectively. As stated in Chapter 2, the average probability of bit error rate, $\operatorname{Pr}(e)=\gamma \operatorname{Pr}($ eljammed $)+$ $(1-\gamma) \operatorname{Pr}(e \mid$ unjammed $)$, is used. It is observed in Figure 5 that there is a worst-jamming fraction $\gamma_{W O R S T}$ for a given $E_{b} / N_{J}$, e.g., $\gamma_{W O R S T}=0.1$ when $E_{b} / N_{J}=10 \mathrm{~dB}$. A jammer can employ this worst-jamming fraction. 


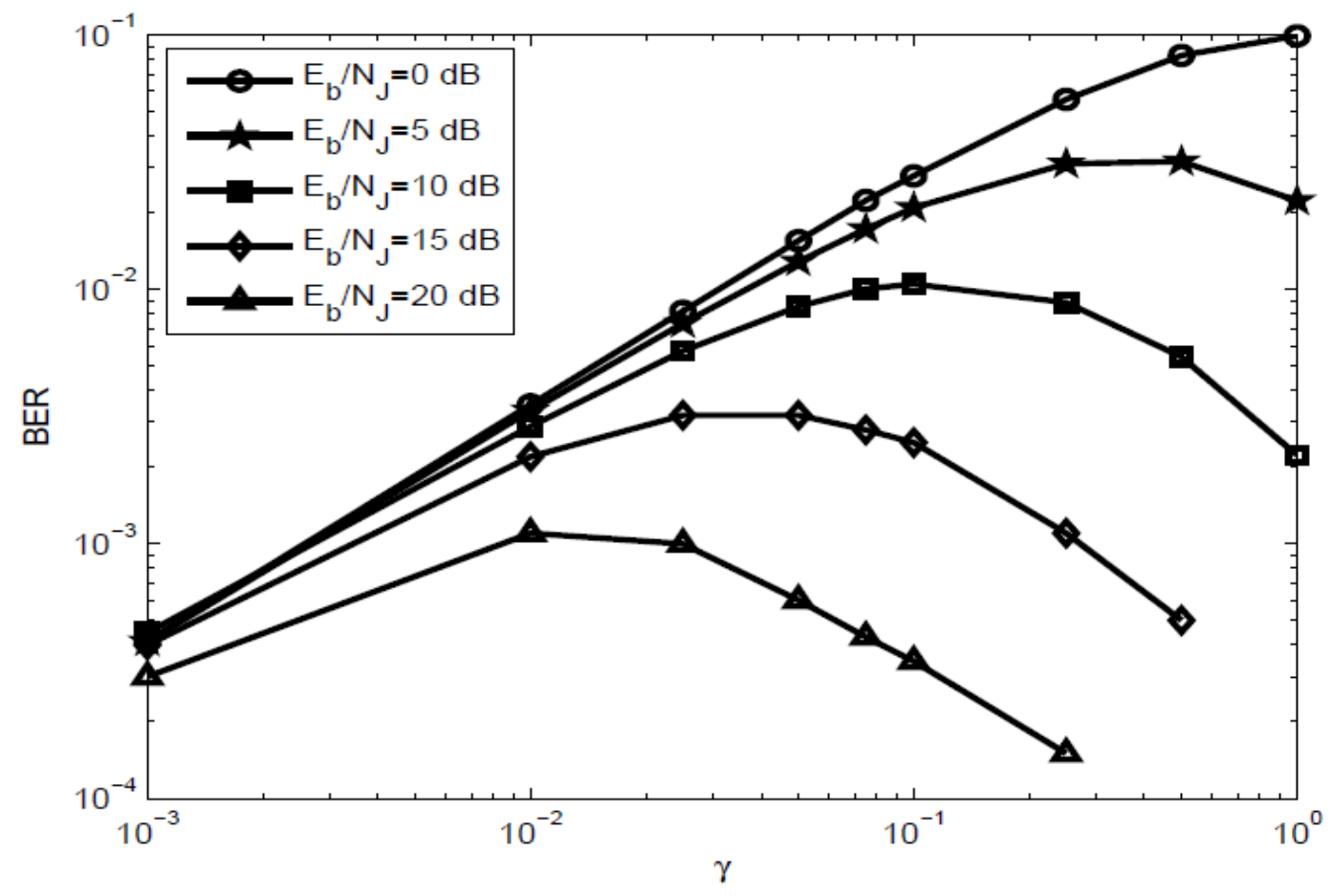

Figure 5. BER versus PBNJ jamming fraction $\gamma$ with different $E_{b} / N_{J}$, i.e., $0,5,10,15,20 \mathrm{~dB}$, when $E_{b} / N_{J}=13 \mathrm{~dB}$ for cooperative $\mathrm{AF} M N L$ wireless relay network under transmit power constraints using $N=6, M=2$, and $L=2$, respectively. 


\section{CHAPTER 6}

\section{CONCLUSIONS}

This thesis investigated cooperative AF $M N L$ wireless relay networks under a jamming environment with various power constraints to determine the optimal precode matrix at the sources and amplifying matrix at the relays based on the MMSE criterion. It was observed that the gain of diversity order can occur as $N$ increases in wireless relay networks, while the diversity order can suffer loss as the jamming signal power increases. It was also found that the best BER performance among power constraint cases proposed in this thesis is observed when the transmit power at both the sources and the relays is constrained during data transmission. Finally, using the proposed system model, the worst jamming fraction $\gamma$ was identified. 
REFERENCES 


\section{LIST OF REFERENCES}

1. B. Wang, J. Zhang, and A. H. Madsen, "On the capacity of MIMO relay channels," IEEE Transactions on Information Theory, vol. 51, no. 1, pp. 29-43, Jan. 2005.

2. S. Yang and J.-C. Belfiore, "Optimal space-time codes for the MIMO amplify-and-forward cooperative channel," IEEE Transactions on Information Theory, vol. 53, no. 2, pp. 647663, Feb. 2007.

3. S. W. Peters and R. W. Heath, Jr., "Nonregenerative MIMO relalying with optimal transmit antenna selection," IEEE Signal Processing Letter, vol. 15, pp. 421-424, 2008.

4. X. Tang and Y. Hua, "Optimal design of non-regenerative MIMO wireless relays," IEEE Transactions Wireless Communication, vol. 6, no. 4, pp. 1398-1407, April 2007.

5. W. Guan and H. Luo, "Joint MMSE transceiver design in nonregenerative MIMO relay systems," IEEE Communication Letters, vol. 12, no.7, pp. 517-519, July 2008.

6. J. Joung and A. H. Sayed, "Design of half- and full-duplex relay systems based on the MMSE formulation," Proceedings of IEEE SSP, Wales, UK, Aug. 2009.

7. J. Joung and A. H. Sayed, "Power allocation for beamformaing relay networks under channel unceratinties," Proceedings of IEEE GLOBE- COM, Honolulu, HI, Nov. 2009.

8. S. Berger and A. Wittneben, "Cooperative distributed multiuser MMSE relaying in wireless ad-hoc networks," Proceedings of IEEE 39th Asilomar Conference, Oct. 2005, pp. 1072-1076.

9. R. Krishna, Z. Xiong, and S. Lambotharan, "A cooperative MMSE relay strategy for wireless sensor networks," IEEE Signal Processing Letters, vol. 15, pp. 549-552, July 2008

10. K. Lee, H. M. Kwon, H. Kim, H. Park, and Y. H. Lee, "Cooperative AF MIMO Wireless relay networks under relay power constraint," Asilomar Conference, Pacific Grove, CA, Nov. 2012.

11. H. Kim, H. M. Kwon, K. Lee, Y. Shim, H. Park, and Y. H. Lee, "Optimal amplify-andforward precode and relay amplifying matrices," IEEE 77th VTC, Dresden, Germany, June 2013.

12. A. S. Behbahani, R. Merched, and A. M. Eltawil, "Optimizations of MIMO relay networks," IEEE Transactions on Signal Processing, vol. 55, no. 7, pp. 3336-3348, July 2007. 


\section{LIST OF REFERENCES (continued)}

13. K. Lee, H. M. Kwon, H. Kim, E. M. Sawan, H. Park, and Y. H. Lee, "AF MIMO wireless relay networks under received power constraint," IEEE VTC 2012 Fall, Quebec, Canada, Sept. 2012.

14. H. Hu and N. Wei, "A study of GPS jamming and anti-jamming," PEITS, Shenzhen, China, Dec. 2009.

15. J. S. Lee, R. H. French, and L. E. Miller, "Probability of error analyses of a BFSK frequency-hopping system with diversity under partial-band jamming interference-Part I: Performance of square-law linear combining soft decision receiver," IEEE Transactions on Communications, vol. COM-32, no. 6, pp. 645-653, June 1984.

16. J. S. Lee, R. H. French, and L. E. Miller, "Error-correcting codes and nonlinear diversity combining against worst case partial-band noise jamming of frequency-hopping MFSK systems," IEEE Transactions on Communications, vol. 36, no. 4, pp. 471-478, April 1988.

17. S. Boyd and L. Vandenberghe, Convex Optimization, Cambridge, UK: Cambridge University Press, 1985.

18. A. Hjørungnes and D. Gesbert, "Complex-valued matrix differentiation: Techniques and key results," IEEE Transactions on Signal Processing, vol. 55, no. 6, pp. 2740-2746, May 2007.

19. J. R. Schott, Matrix Analysis for Statistics, 2nd ed., Hoboken, NJ: John Wiley \& Sons, Inc., 2005.

20. F. L. Lewis and V. L. Syrmos, Optimal Control, 2nd ed., New York, NY: John Wiley \& Sons, Inc., 1995. 
APPENDIX 


\section{APPENDIX}

\section{DERIVATION OF PRECODE MATRIX W AND AMPLIFYING MATRIX F}

This appendix derives the optimum precode matrix $\mathbf{W}$ at the sources and amplifying matrix $\mathbf{F}$ at the relays under transmit power constraints case. The derivation of other power constraints cases are very similar to the transmit power constraints case. Therefore, the details are omitted.

$$
\begin{aligned}
\mathbf{r} & =\mathbf{H}_{\mathrm{s}} \mathbf{d}+\mathbf{v}_{\mathrm{s}}+\mathbf{j}_{1} \cdot \\
\mathbf{x} & =\mathbf{F r} \\
& =\mathbf{F}\left(\mathbf{H}_{\mathrm{s}} \mathbf{W} \mathbf{s}+\mathbf{v}_{\mathrm{s}}+\mathbf{j}_{1}\right) \\
& =\mathbf{F} \mathbf{H}_{\mathrm{s}} \mathbf{W} \mathbf{s}+\mathbf{F} \mathbf{v}_{\mathrm{s}}+\mathbf{F} \mathbf{j}_{1} . \\
\mathbf{y} & =\mathbf{H}_{\mathrm{y}} \mathbf{x}+\mathbf{v}_{\mathrm{y}}+\mathbf{j}_{2} \\
& =\mathbf{H}_{\mathrm{y}}\left(\mathbf{F} \mathbf{H}_{\mathrm{s}} \mathbf{W} \mathbf{s}+\mathbf{F} \mathbf{v}_{\mathrm{s}}+\mathbf{F j}_{1}\right)+\mathbf{v}_{\mathrm{y}}+\mathbf{j}_{2} \\
& =\mathbf{H}_{\mathrm{y}} \mathbf{F} \mathbf{H}_{\mathrm{s}} \mathbf{W} \mathbf{s}+\mathbf{H}_{\mathrm{y}} \mathbf{F} \mathbf{v}_{\mathrm{s}}+\mathbf{H}_{\mathrm{y}} \mathbf{F} \mathbf{j}_{1}+\mathbf{j}_{2}+\mathbf{v}_{\mathrm{y}} .
\end{aligned}
$$

$$
\begin{aligned}
J(\mathbf{W}, \mathbf{F}, \alpha) \triangleq & E\left[\|\mathbf{s}-\hat{\mathbf{y}}\|^{2}\right] \\
= & E\left[\operatorname{tr}\left[(\mathbf{s}-\hat{\mathbf{y}})(\mathbf{s}-\hat{\mathbf{y}})^{H}\right]\right] \\
= & E\left[\operatorname { t r } \left[\left(\alpha^{-1} \mathbf{H}_{\mathrm{y}} \mathbf{F} \mathbf{H}_{\mathrm{s}} \mathbf{W} \mathbf{s}+\alpha^{-1} \mathbf{H}_{\mathrm{y}} \mathbf{F} \mathbf{v}_{\mathrm{s}}+\alpha^{-1} \mathbf{H}_{\mathrm{y}} \mathbf{F} \mathbf{j}_{1}+\alpha^{-1} \mathbf{v}_{\mathrm{y}}+\alpha^{-1} \mathbf{j}_{2}-\mathbf{s}\right)\right.\right. \\
& \left.\left.\quad \times\left(\alpha^{-1} \mathbf{s}^{H} \mathbf{W}^{H} \mathbf{H}_{\mathrm{s}}^{H} \mathbf{F}^{H} \mathbf{H}_{\mathrm{y}}^{H}+\alpha^{-1} \mathbf{v}_{\mathrm{s}}^{H} \mathbf{F}^{H} \mathbf{H}_{\mathrm{y}}^{H}+\alpha^{-1} \mathbf{j}_{1}^{H} \mathbf{F}^{H} \mathbf{H}_{\mathrm{y}}^{H}+\alpha^{-1} \mathbf{v}_{\mathrm{y}}^{H}+\alpha^{-1} \mathbf{j}_{2}^{H}-\mathbf{s}^{H}\right)\right]\right] \\
= & E\left[\operatorname { t r } \left[\alpha^{-2} \mathbf{H}_{\mathrm{y}} \mathbf{F} \mathbf{H}_{\mathrm{s}} \mathbf{W} \mathbf{s} \mathbf{s}^{H} \mathbf{W}^{H} \mathbf{H}_{\mathrm{s}}^{H} \mathbf{F}^{H} \mathbf{H}_{\mathrm{y}}^{H}+\alpha^{-2} \mathbf{H}_{\mathrm{y}} \mathbf{F} \mathbf{v}_{\mathrm{s}} \mathbf{v}_{\mathrm{s}}^{H} \mathbf{F}^{H} \mathbf{H}_{\mathrm{y}}^{H}+\alpha^{-2} \mathbf{H}_{\mathrm{y}} \mathbf{F} \mathbf{j}_{1} \mathbf{j}_{1}^{H} \mathbf{F}^{H} \mathbf{H}_{\mathrm{y}}^{H}\right.\right. \\
& \left.\left.\quad+\alpha^{-1} \mathbf{v}_{\mathrm{y}} \mathbf{v}_{\mathrm{y}}^{H}+\alpha^{-1} \mathbf{j}_{2} \mathbf{j}_{2}^{H}+\mathbf{s} \mathbf{s}^{H}-\alpha^{-1} \mathbf{H}_{\mathrm{y}} \mathbf{F} \mathbf{H}_{\mathrm{s}} \mathbf{W} \mathbf{s} \mathbf{s}^{H}-\alpha^{-1} \mathbf{s s}^{H} \mathbf{W}^{H} \mathbf{H}_{\mathrm{s}}^{H} \mathbf{F}^{H} \mathbf{H}_{\mathrm{y}}^{H}\right]\right] \\
= & \alpha^{-2} \sigma_{s}{ }^{2}\left\|\mathbf{H}_{\mathrm{y}} \mathbf{F} \mathbf{H}_{\mathrm{s}} \mathbf{W}\right\|_{F}^{2}+\alpha^{-2} \beta\left\|\mathbf{H}_{\mathrm{y}} \mathbf{F}\right\|_{F}^{2}+M \sigma_{s}{ }^{2}-2 \alpha^{-1} \sigma_{s}{ }^{2} \operatorname{tr}\left(\operatorname{Re}\left[\mathbf{H}_{\mathrm{y}} \mathbf{F} \mathbf{H}_{\mathrm{s}} \mathbf{W}\right]\right) \\
& +\alpha^{-2} \omega L .
\end{aligned}
$$


APPENDIX (continued)

where $\hat{\mathbf{y}}=\alpha^{-1} \mathbf{y}, \beta={\sigma_{v_{s}}}{ }^{2}+{\sigma_{j_{1}}}^{2}, \omega={\sigma_{v_{y}}}^{2}+{\sigma_{j_{2}}}^{2}$

$\mathrm{P}_{\mathrm{s}}=E\left[\|\mathbf{W} \mathbf{s}\|^{2}\right]=\operatorname{tr}\left(\mathbf{W} \mathbf{s} \mathbf{s}^{H} \mathbf{W}^{H}\right)=\sigma_{s}{ }^{2}\|\mathbf{W}\|_{F}^{2}$.

$$
\begin{aligned}
\mathrm{P}_{\mathrm{R}}=E\left[\|\mathbf{x}\|^{2}\right] & =\operatorname{tr}\left[\left(\mathbf{F} \mathbf{H}_{\mathbf{s}} \mathbf{W} \mathbf{s}+\mathbf{F} \mathbf{v}_{\mathbf{s}}+\mathbf{F} \mathbf{j}_{1}\right)\left(\mathbf{s}^{H} \mathbf{W}^{H} \mathbf{H}_{\mathrm{s}}^{H} \mathbf{F}^{H}+\mathbf{v}_{\mathbf{s}}^{H} \mathbf{F}^{H}+\mathbf{j}_{1}^{H} \mathbf{F}^{H}\right)\right] \\
& =\sigma_{s}{ }^{2}\left\|\mathbf{F} \mathbf{H}_{\mathrm{s}} \mathbf{W}\right\|_{F}^{2}+\left({\sigma_{v_{s}}}^{2}+{\sigma_{j_{1}}}^{2}\right)\|\mathbf{F}\|_{F}^{2} \\
& =\sigma_{s}{ }^{2}\left\|\mathbf{F} \mathbf{H}_{\mathrm{s}} \mathbf{W}\right\|_{F}^{2}+\beta\|\mathbf{F}\|_{F}^{2} .
\end{aligned}
$$

$$
\begin{aligned}
P_{Y}=E\left[\|\mathbf{y}\|^{2}\right]= & \operatorname{tr}\left[\left(\mathbf{H}_{\mathrm{y}} \mathbf{F} \mathbf{H}_{\mathrm{s}} \mathbf{W} \mathbf{s}+\mathbf{H}_{\mathrm{y}} \mathbf{F} \mathbf{v}_{\mathrm{s}}+\mathbf{H}_{\mathrm{y}} \mathbf{F} \mathbf{j}_{1}+\mathbf{v}_{\mathbf{y}}+\mathbf{j}_{2}\right)\right. \\
& \times\left(\mathbf{s}^{H} \mathbf{W}^{H} \mathbf{H}_{\mathrm{s}}^{H} \mathbf{F}^{H} \mathbf{H}_{\mathrm{y}}^{H}+\mathbf{v}_{\mathrm{s}}^{H} \mathbf{F}^{H} \mathbf{H}_{\mathrm{y}}^{H}+\mathbf{j}_{1}^{H} \mathbf{F}^{H} \mathbf{H}_{\mathrm{y}}^{H}+\mathbf{v}_{\mathrm{y}}^{H}+\mathbf{j}_{2}^{H}\right) \\
= & \sigma_{\mathrm{s}}{ }^{2}\left\|\mathbf{H}_{\mathrm{y}} \mathbf{F} \mathbf{H}_{\mathrm{s}} \mathbf{W}\right\|_{F}^{2}+\beta\left\|\mathbf{H}_{\mathrm{y}} \mathbf{F}\right\|_{F}^{2}+\omega .
\end{aligned}
$$

$L\left(\mathbf{W}, \mathbf{F}, \alpha, \lambda_{s}, \lambda_{R}\right)=J(\mathbf{W}, \mathbf{F}, \alpha)+\lambda_{\mathrm{s}}\left(E\left[\|\mathbf{d}\|^{2}\right]-\mathrm{P}_{\mathrm{s}}\right)+\lambda_{\mathrm{R}}\left(E\left[\|\mathbf{x}\|^{2}\right]-\mathrm{P}_{\mathrm{R}}\right)$

$$
\begin{aligned}
= & \alpha^{-2}{\sigma_{s}}^{2}\left\|\mathbf{H}_{\mathrm{y}} \mathbf{F} \mathbf{H}_{\mathrm{s}} \mathbf{W}\right\|_{F}^{2}+\alpha^{-2} \beta\left\|\mathbf{H}_{\mathrm{y}} \mathbf{F}\right\|_{F}^{2}+M{\sigma_{s}}^{2} \\
& -2 \alpha^{-1}{\sigma_{s}}^{2} \operatorname{tr}\left(\operatorname{Re}\left[\mathbf{H}_{\mathrm{y}} \mathbf{F} \mathbf{H}_{\mathrm{s}} \mathbf{W}\right]\right)+\alpha^{-2} \omega L \\
& +\lambda_{\mathrm{s}}\left({\sigma_{s}}^{2} \operatorname{tr}\left(\mathbf{W} \mathbf{W}^{H}\right)-\mathrm{P}_{\mathrm{s}}\right) \\
& \left.+\lambda_{\mathrm{R}}\left(\sigma_{s}{ }^{2} \operatorname{tr}\left(\mathbf{F} \mathbf{H}_{\mathrm{s}} \mathbf{W} \mathbf{W}^{H} \mathbf{H}_{\mathrm{s}}^{H} \mathbf{F}^{H}\right)+\beta \operatorname{tr}\left(\mathbf{F F}^{H}\right)\right)-\mathrm{P}_{\mathrm{R}}\right) .
\end{aligned}
$$

$$
\begin{aligned}
\frac{\partial L(\mathbf{F}, \lambda)}{\partial \mathbf{F}^{*}}= & \alpha^{-2} \sigma_{s}{ }^{2} \mathbf{H}_{\mathrm{y}}^{H} \mathbf{H}_{\mathrm{y}} \mathbf{F} \mathbf{H}_{\mathrm{s}} \mathbf{W} \mathbf{W}^{H} \mathbf{H}_{\mathrm{s}}^{H}+\alpha^{-2} \beta \mathbf{H}_{\mathrm{y}}^{H} \mathbf{H}_{\mathrm{y}} \mathbf{F}-\alpha^{-1}{\sigma_{s}}^{2} \mathbf{H}_{\mathrm{y}}^{H} \mathbf{W}^{H} \mathbf{H}_{\mathrm{s}}^{H} \\
& +\lambda_{\mathrm{R}} \sigma_{s}{ }^{2} \mathbf{F} \mathbf{H}_{\mathrm{s}} \mathbf{W} \mathbf{W}^{H} \mathbf{H}_{\mathrm{s}}^{H}+\beta \lambda_{\mathrm{R}} \mathbf{F}=\mathbf{0}_{N \times N} .
\end{aligned}
$$

$$
\begin{aligned}
\frac{\partial L(\mathbf{F}, \lambda)}{\partial \mathbf{W}^{*}}= & \alpha^{-2} \sigma_{s}{ }^{2} \mathbf{H}_{\mathrm{s}}^{H} \mathbf{F}^{H} \mathbf{H}_{\mathrm{y}}^{H} \mathbf{H}_{\mathrm{y}} \mathbf{F} \mathbf{H}_{\mathrm{s}} \mathbf{W}-\alpha^{-1} \sigma_{s}{ }^{2} \mathbf{H}_{\mathrm{s}}^{H} \mathbf{F}^{H} \mathbf{H}_{\mathrm{y}}^{H}+\lambda_{\mathrm{s}} \sigma_{s}{ }^{2} \mathbf{W} \\
& +\lambda_{\mathrm{R}} \sigma_{s}{ }^{2} \mathbf{H}_{\mathrm{s}}^{H} \mathbf{F}^{H} \mathbf{F} \mathbf{H}_{\mathrm{s}} \mathbf{W}=\mathbf{0}_{M \times M} .
\end{aligned}
$$


APPENDIX (continued)

$$
\begin{aligned}
& \frac{\partial L(\mathbf{F}, \lambda)}{\partial \alpha}= 2 \alpha^{-2} \sigma_{s}^{2} \operatorname{tr}\left(\operatorname{Re}\left[\mathbf{H}_{\mathrm{y}} \mathbf{F} \mathbf{H}_{\mathrm{s}} \mathbf{W}\right]\right)-2 \alpha^{-3} \beta\left\|\mathbf{H}_{\mathrm{y}} \mathbf{F}\right\|_{F}^{2}-2 \alpha^{-3} \sigma_{s}^{2}\left\|\mathbf{H}_{\mathrm{y}} \mathbf{F} \mathbf{H}_{\mathrm{s}} \mathbf{W}\right\|_{F}^{2} \\
&-2 \alpha^{-3} \omega L=0 . \\
& \frac{\partial L(\mathbf{F}, \lambda)}{\partial \lambda_{s}}=\sigma_{s}^{2}\|\mathbf{W}\|_{F}^{2}-\mathrm{Ps}=0 . \\
& \frac{\partial L(\mathbf{F}, \lambda)}{\partial \lambda_{\mathrm{R}}}=\sigma_{s}^{2}\left\|\mathbf{F} \mathbf{H}_{\mathrm{s}} \mathbf{W}\right\|_{F}^{2}+\beta\|\mathbf{F}\|_{F}^{2}-\mathrm{P}_{\mathrm{R}}=0 .
\end{aligned}
$$

Multiplying $\alpha^{2}$ to equation (A9) yields

$$
\begin{aligned}
\sigma_{s}^{2} \mathbf{H}_{\mathrm{y}}^{H} \mathbf{H}_{\mathrm{y}} \mathbf{F} \mathbf{H}_{\mathrm{s}} \mathbf{W} \mathbf{W}^{H} \mathbf{H}_{\mathrm{s}}^{H}+\beta \mathbf{H}_{\mathrm{y}}^{H} \mathbf{H}_{\mathrm{y}} \mathbf{F}+\alpha^{2} \lambda_{\mathrm{R}} \mathbf{F}\left(\sigma_{s}{ }^{2} \mathbf{H}_{\mathrm{s}} \mathbf{W} \mathbf{W}^{H} \mathbf{H}_{\mathrm{s}}^{H}+\beta \mathbf{I}_{N}\right)=\alpha \sigma_{s}{ }^{2} \mathbf{H}_{\mathrm{y}}^{H} \mathbf{W}^{H} \mathbf{H}_{\mathrm{s}}^{H} \\
\Rightarrow\left(\mathbf{H}_{\mathrm{y}}^{H} \mathbf{H}_{\mathrm{y}}+\alpha^{2} \lambda_{\mathrm{R}} \mathbf{I}_{N}\right) \mathbf{F}\left(\sigma_{s}^{2} \mathbf{H}_{\mathrm{s}} \mathbf{W} \mathbf{W}^{H} \mathbf{H}_{\mathrm{s}}^{H}+\beta \mathbf{I}_{N}\right)=\alpha \sigma_{s}{ }^{2} \mathbf{H}_{\mathrm{y}}^{H} \mathbf{W}^{H} \mathbf{H}_{\mathrm{s}}^{H} \\
\Rightarrow \mathbf{F}^{\dagger}=\left(\mathbf{H}_{\mathrm{y}}^{H} \mathbf{H}_{\mathrm{y}}+\alpha^{2} \lambda_{\mathrm{R}} \mathbf{I}_{N}\right)^{-1}\left(\alpha \sigma_{s}{ }^{2} \mathbf{H}_{\mathrm{y}}^{H} \mathbf{W}^{H} \mathbf{H}_{\mathrm{s}}^{H}\right)\left(\sigma_{s}{ }^{2} \mathbf{H}_{\mathrm{s}} \mathbf{W} \mathbf{W}^{H} \mathbf{H}_{\mathrm{s}}^{H}+\beta \mathbf{I}_{N}\right)^{-1} \\
\quad=\left(\mathbf{H}_{\mathrm{y}}^{H} \mathbf{H}_{\mathrm{y}}+\alpha^{2} \lambda_{\mathrm{R}} \mathbf{I}_{N}\right)^{-1} \mathbf{H}_{\mathrm{a}} \mathbf{H}_{\mathrm{b}}^{-1} \alpha \sigma_{s}{ }^{2} \\
\quad=\mathbf{H}_{\mathrm{t}}^{-1} \mathbf{H}_{\mathrm{a}} \mathbf{H}_{\mathrm{b}}^{-1} \alpha \sigma_{s}{ }^{2}
\end{aligned}
$$

Multiplying $\alpha^{2} \mathbf{F}^{H}$ to equation (A9) and then applying trace yields

$$
\begin{aligned}
& \sigma_{s}{ }^{2} \mathbf{H}_{\mathrm{y}}^{H} \mathbf{H}_{\mathrm{y}} \mathbf{F} \mathbf{H}_{\mathrm{s}} \mathbf{W} \mathbf{W}^{H} \mathbf{H}_{\mathrm{s}}^{H} \mathbf{F}^{H}+\beta \mathbf{H}_{\mathrm{y}}^{H} \mathbf{H}_{\mathrm{y}} \mathbf{F} \mathbf{F}^{H}-\alpha \sigma_{s}{ }^{2} \mathbf{H}_{\mathrm{y}}^{H} \mathbf{W}^{H} \mathbf{H}_{\mathrm{s}}^{H} \mathbf{F}^{H}+\alpha^{2} \lambda_{\mathrm{R}} \sigma_{s}{ }^{2} \mathbf{F} \mathbf{H}_{\mathrm{s}} \mathbf{W} \mathbf{W}^{H} \mathbf{H}_{\mathrm{s}}^{H} \mathbf{F}^{H} \\
& +\alpha^{2} \beta \lambda_{\mathrm{R}} \mathbf{F} \mathbf{F}^{H}=\mathbf{0}_{N \times N} \\
& \Rightarrow \sigma_{s}{ }^{2}\left\|\mathbf{H}_{\mathrm{y}} \mathbf{F} \mathbf{H}_{\mathrm{s}} \mathbf{W}\right\|_{F}^{2}+\beta\left\|\mathbf{H}_{\mathrm{y}} \mathbf{F}\right\|_{F}^{2}+\alpha^{2} \lambda_{\mathrm{R}}\left(\sigma_{s}{ }^{2}\left\|\mathbf{F} \mathbf{H}_{\mathrm{s}} \mathbf{W}\right\|_{F}^{2}+\beta\|\mathbf{F}\|_{F}^{2}\right) \\
& \quad=\alpha \sigma_{s}{ }^{2} \operatorname{tr}\left(\mathbf{H}_{\mathrm{y}}^{H} \mathbf{W}^{H} \mathbf{H}_{\mathrm{s}}^{H} \mathbf{F}^{H}\right) \\
& \Rightarrow \sigma_{s}{ }^{2}\left\|\mathbf{H}_{\mathrm{y}} \mathbf{F} \mathbf{H}_{\mathrm{s}} \mathbf{W}\right\|_{F}^{2}+\beta\left\|\mathbf{H}_{\mathrm{y}} \mathbf{F}\right\|_{F}^{2}+\alpha^{2} \lambda_{\mathrm{R}} \mathrm{P}_{\mathrm{R}}=\alpha \sigma_{s}{ }^{2} \operatorname{tr}\left(\mathbf{H}_{\mathrm{y}}^{H} \mathbf{W}^{H} \mathbf{H}_{\mathrm{s}}^{H} \mathbf{F}^{H}\right)
\end{aligned}
$$


Multiplying $-\frac{1}{2} \alpha^{3}$ to equation (A11) yields

$\beta\left\|\mathbf{H}_{\mathrm{y}} \mathbf{F}\right\|_{F}^{2}+\sigma_{s}^{2}\left\|\mathbf{H}_{\mathrm{y}} \mathbf{F} \mathbf{H}_{\mathrm{s}} \mathbf{W}\right\|_{F}^{2}+\omega L=\alpha \sigma_{s}{ }^{2} \operatorname{tr}\left(\mathbf{H}_{\mathrm{y}} \mathbf{F} \mathbf{H}_{\mathrm{s}} \mathbf{W}\right)$.

From equations (A15) and (A16),

$$
\begin{aligned}
& \alpha^{2} \lambda_{\mathrm{R}} \mathrm{P}_{\mathrm{R}}=\omega L \\
& \Rightarrow \alpha^{2} \lambda_{\mathrm{R}}=\frac{\omega L}{\mathrm{P}_{\mathrm{R}}} .
\end{aligned}
$$

Applying equation (A14) to equation (A6) yields

$$
\begin{aligned}
\mathrm{P}_{\mathrm{R}} & =\sigma_{s}^{2}\left\|\mathbf{F H}_{\mathrm{s}} \mathbf{W}\right\|_{F}^{2}+\beta\|\mathbf{F}\|_{F}^{2} \\
& =\alpha^{2} \sigma_{s}{ }^{4}\left(\left\|\sigma_{s}^{2} \mathbf{H}_{\mathrm{t}}^{-1} \mathbf{H}_{\mathrm{a}} \mathbf{H}_{\mathrm{b}}^{-1} \mathbf{H}_{\mathrm{s}} \mathbf{W}\right\|_{F}^{2}+\beta\left\|\mathbf{H}_{\mathrm{t}}^{-1} \mathbf{H}_{\mathrm{a}} \mathbf{H}_{\mathrm{b}}^{-1}\right\|_{F}^{2}\right) .
\end{aligned}
$$

From equation (A18),

$$
\alpha^{\dagger}=\frac{\sigma_{s}^{-2} \sqrt{\mathrm{P}_{\mathrm{R}}}}{\sqrt{\sigma_{s}^{2}\left\|\mathbf{H}_{\mathrm{t}}^{-1} \mathbf{H}_{\mathrm{a}} \mathbf{H}_{\mathrm{b}}^{-1} \mathbf{H}_{\mathrm{s}} \mathbf{W}\right\|_{F}^{2}+\beta\left\|\mathbf{H}_{\mathrm{t}}^{-1} \mathbf{H}_{\mathrm{a}} \mathbf{H}_{\mathrm{b}}^{-1}\right\|_{F}^{2}}} .
$$

Applying equation (A19) to equation (A14) yields

$$
\begin{aligned}
\mathbf{F}^{\dagger} & =\mathbf{H}_{\mathrm{t}}^{-1} \mathbf{H}_{\mathrm{a}} \mathbf{H}_{\mathrm{b}}^{-1} \alpha \sigma_{s}^{2} \\
& =\sqrt{\frac{\mathrm{P}_{\mathrm{R}}}{\sigma_{\mathrm{s}}^{2}\left\|\mathbf{H}_{\mathrm{t}}^{-1} \mathbf{H}_{\mathrm{a}} \mathbf{H}_{\mathrm{b}}^{-1} \mathbf{H}_{\mathrm{s}} \mathbf{W}\right\|_{F}^{2}+\beta\left\|\mathbf{H}_{\mathrm{t}}^{-1} \mathbf{H}_{\mathrm{a}} \mathbf{H}_{\mathrm{b}}^{-1}\right\|_{F}^{2}}} \times \mathbf{H}_{\mathrm{t}}^{-1} \mathbf{H}_{\mathrm{a}} \mathbf{H}_{\mathrm{b}}^{-1} .
\end{aligned}
$$

Applying equation (A19) to equation (A17) yields

$$
\begin{aligned}
\lambda_{\mathrm{R}}^{\dagger} & =\alpha^{-2} L \omega \mathrm{P}_{\mathrm{R}}^{-1} \\
& =\sigma_{s}^{4} L \omega \mathrm{P}_{\mathrm{R}}^{-2}\left(\sigma_{s}^{2}\left\|\mathbf{H}_{\mathrm{t}}^{-1} \mathbf{H}_{\mathrm{a}} \mathbf{H}_{\mathrm{b}}^{-1} \mathbf{H}_{\mathrm{s}} \mathbf{W}\right\|_{F}^{2}+\beta\left\|\mathbf{H}_{\mathrm{t}}^{-1} \mathbf{H}_{\mathrm{a}} \mathbf{H}_{\mathrm{b}}^{-1}\right\|_{F}^{2}\right) .
\end{aligned}
$$


Multiplying $\alpha^{2}$ to equation (A10) yields

$$
\begin{aligned}
\left(\mathbf{H}_{\mathrm{s}}^{H} \mathbf{F}^{H} \mathbf{H}_{\mathrm{y}}^{H} \mathbf{H}_{\mathrm{y}} \mathbf{F} \mathbf{H}_{\mathrm{s}}+\alpha^{2} \lambda_{\mathrm{R}} \mathbf{H}_{\mathrm{s}}^{H} \mathbf{F}^{H} \mathbf{F} \mathbf{H}_{\mathrm{s}}+\alpha^{2} \lambda_{\mathrm{s}} \mathbf{I}_{M}\right) \mathbf{W}=\alpha \mathbf{H}_{\mathrm{s}}^{H} \mathbf{F}^{H} \mathbf{H}_{\mathrm{y}}^{H} \\
\Rightarrow \mathbf{W}=\alpha\left(\mathbf{H}_{\mathrm{s}}^{H} \mathbf{F}^{H} \mathbf{H}_{\mathrm{y}}^{H} \mathbf{H}_{\mathrm{y}} \mathbf{F} \mathbf{H}_{\mathrm{s}}+\alpha^{2} \lambda_{\mathrm{R}} \mathbf{H}_{\mathrm{s}}^{H} \mathbf{F}^{H} \mathbf{F} \mathbf{H}_{\mathrm{s}}+\alpha^{2} \lambda_{\mathrm{s}} \mathbf{I}_{M}\right)^{-1} \mathbf{H}_{\mathrm{s}}^{H} \mathbf{F}^{H} \mathbf{H}_{\mathrm{y}}^{H} \\
=\alpha\left(\mathbf{H}_{\mathrm{c}}+\mathbf{H}_{\mathrm{d}}+\alpha^{2} \lambda_{\mathrm{s}} \mathbf{I}_{M}\right)^{-1} \mathbf{H}_{\mathrm{s}}^{H} \mathbf{F}^{H} \mathbf{H}_{\mathrm{y}}^{H} \\
=\alpha \mathbf{H}_{\mathrm{k}}^{-1} \mathbf{H}_{\mathrm{s}}^{H} \mathbf{F}^{H} \mathbf{H}_{\mathrm{y}}^{H} .
\end{aligned}
$$

Applying equation (A21) to equation( A5) yields

$$
\begin{aligned}
\mathrm{P}_{\mathrm{s}} & =\sigma_{s}^{2}\|\mathbf{W}\|_{F}^{2} \\
& =\sigma_{s}{ }^{2}\left\|\alpha \mathbf{H}_{\mathrm{k}}^{-1} \mathbf{H}_{\mathrm{s}}^{H} \mathbf{F}^{H} \mathbf{H}_{\mathrm{y}}^{H}\right\|_{F}^{2} .
\end{aligned}
$$

From equation (A22),

$$
\alpha=\sqrt{\frac{\mathrm{P}_{\mathrm{s}}}{\sigma_{\mathrm{s}}{ }^{2}\left\|\mathbf{H}_{\mathrm{k}}^{-1} \mathbf{H}_{\mathrm{s}}^{H} \mathbf{F}^{H} \mathbf{H}_{\mathrm{y}}^{H}\right\|_{F}^{2}}} .
$$

Applying equation (A23) to equation (A21) yields

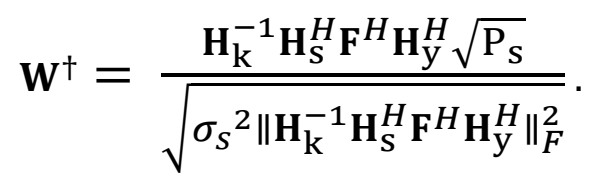

From equation (A16),

$\alpha \sigma_{s}{ }^{2} \operatorname{tr}\left(\mathbf{H}_{\mathrm{y}} \mathbf{F} \mathbf{H}_{\mathrm{s}} \mathbf{W}\right)=\beta\left\|\mathbf{H}_{\mathrm{y}} \mathbf{F}\right\|_{F}^{2}+\sigma_{\mathrm{s}}{ }^{2}\left\|\mathbf{H}_{\mathrm{y}} \mathbf{F} \mathbf{H}_{\mathrm{s}} \mathbf{W}\right\|_{F}^{2}+\omega L$.

Multiplying $\alpha^{2} \mathbf{W}^{H}$ to (A10) and then applying trace yields $\operatorname{tr}\left(\sigma_{s}{ }^{2} \mathbf{H}_{\mathrm{s}}^{H} \mathbf{F}^{H} \mathbf{H}_{\mathrm{y}}^{H} \mathbf{H}_{\mathrm{y}} \mathbf{F} \mathbf{H}_{\mathrm{s}} \mathbf{W} \mathbf{W}^{H}-\alpha \sigma_{\mathrm{s}}{ }^{2} \mathbf{H}_{\mathrm{s}}^{H} \mathbf{F}^{H} \mathbf{H}_{\mathrm{y}}^{H} \mathbf{W}^{H}+\alpha^{2} \lambda_{\mathrm{s}} \sigma_{s}{ }^{2} \mathbf{W} \mathbf{W}^{H}\right.$ 


$$
\begin{aligned}
\left.+\alpha^{2} \lambda_{\mathrm{R}} \sigma_{s}{ }^{2} \mathbf{H}_{\mathrm{s}}^{H} \mathbf{F}^{H} \mathbf{F} \mathbf{H}_{\mathrm{s}} \mathbf{W} \mathbf{W}^{H}\right)=\mathbf{0}_{M \times M} & \\
\Rightarrow \alpha \sigma_{s}{ }^{2} \operatorname{tr}\left(\mathbf{H}_{\mathrm{s}}^{H} \mathbf{F}^{H} \mathbf{H}_{\mathrm{y}}^{H} \mathbf{W}^{H}\right) & =\sigma_{s}{ }^{2}\left\|\mathbf{H}_{\mathrm{y}} \mathbf{F} \mathbf{H}_{\mathrm{s}} \mathbf{W}\right\|_{F}^{2}+\alpha^{2} \lambda_{\mathrm{s}} \sigma_{s}{ }^{2}\|\mathbf{W}\|_{F}^{2} \\
& +\alpha^{2} \lambda_{\mathrm{R}} \sigma_{s}{ }^{2}\left\|\mathbf{F} \mathbf{H}_{\mathrm{s}} \mathbf{W}\right\|_{F}^{2} .
\end{aligned}
$$

Using equations (A25) and (A26),

$$
\begin{aligned}
& \sigma_{S}^{2}\left\|\mathbf{H}_{\mathrm{y}} \mathbf{F} \mathbf{H}_{\mathrm{s}} \mathbf{W}\right\|_{F}^{2}+\alpha^{2} \lambda_{\mathrm{s}} \sigma_{S}^{2}\|\mathbf{W}\|_{F}^{2}+\alpha^{2} \lambda_{\mathrm{R}} \sigma_{s}^{2}\left\|\mathbf{F} \mathbf{H}_{\mathrm{s}} \mathbf{W}\right\|_{F}^{2} \\
& =\sigma_{s}{ }^{2}\left\|\mathbf{H}_{\mathrm{y}} \mathbf{F} \mathbf{H}_{\mathrm{s}} \mathbf{W}\right\|_{F}^{2}+\beta\left\|\mathbf{H}_{\mathrm{y}} \mathbf{F}\right\|_{F}^{2}+\omega L .
\end{aligned}
$$

From equation (A27),

$$
\lambda_{\mathrm{s}}=\frac{1}{\alpha^{2}} \times \frac{\beta\left\|\mathbf{H}_{\mathrm{y}} \mathbf{F}\right\|_{F}^{2}+\omega L-\frac{\omega L}{\mathrm{P}_{\mathrm{R}}} \sigma_{s}^{2}\left\|\mathbf{F} \mathbf{H}_{\mathrm{s}} \mathbf{W}\right\|_{F}^{2}}{\sigma_{s}^{2}\|\mathbf{W}\|_{F}^{2}} .
$$

From equation (A28),

$\sigma_{S}^{2}\left\|\mathbf{F H}_{\mathrm{s}} \mathbf{W}\right\|_{F}^{2}=\mathrm{P}_{\mathrm{R}}-\beta\|\mathbf{F}\|_{F}^{2}$.

Using equations (29), (A23), (A28), and (A29),

$$
\begin{aligned}
\lambda_{\mathrm{s}}^{\dagger} & =\frac{\sigma_{s}^{2}\left\|\mathbf{H}_{\mathrm{k}}^{-1} \mathbf{H}_{\mathrm{s}}^{H} \mathbf{F}^{H} \mathbf{H}_{\mathrm{y}}^{H}\right\|_{F}^{2}}{\mathrm{P}_{\mathrm{s}}} \times \frac{\beta\left\|\mathbf{H}_{\mathrm{y}} \mathbf{F}\right\|_{F}^{2}+\omega L-\frac{\omega L}{\mathrm{P}_{\mathrm{R}}} \sigma_{s}^{2}\left\|\mathbf{F} \mathbf{H}_{\mathrm{s}} \mathbf{W}\right\|_{F}^{2}}{\sigma_{s}^{2}\|\mathbf{W}\|_{F}^{2}} \\
& =\frac{\sigma_{s}^{2}\left\|\mathbf{H}_{\mathrm{k}}^{-1} \mathbf{H}_{\mathrm{s}}^{H} \mathbf{F}^{H} \mathbf{H}_{\mathrm{y}}^{H}\right\|_{F}^{2}}{\mathrm{P}_{\mathrm{s}}} \times \frac{\beta\left\|\mathbf{H}_{\mathrm{y}} \mathbf{F}\right\|_{F}^{2}+\omega L-\frac{\omega L}{\mathrm{P}_{\mathrm{R}}}\left(\mathrm{P}_{\mathrm{R}}-\beta\|\mathbf{F}\|_{F}^{2}\right)}{\mathrm{P}_{\mathrm{s}}} \\
& =\frac{\sigma_{S}^{2}\left\|\mathbf{H}_{\mathrm{k}}^{-1} \mathbf{H}_{\mathrm{s}}^{H} \mathbf{F}^{H} \mathbf{H}_{\mathrm{y}}^{H}\right\|_{F}^{2}}{\mathrm{P}_{\mathrm{s}}} \times \frac{\beta\left\|\mathbf{H}_{\mathrm{y}} \mathbf{F}\right\|_{F}^{2}-\frac{\omega \beta L}{\mathrm{P}_{\mathrm{R}}}\|\mathbf{F}\|_{F}^{2}}{\mathrm{P}_{\mathrm{S}}} \\
& =\varepsilon \sigma_{\mathrm{s}}{ }^{2} \mathrm{P}_{\mathrm{S}}^{-1}\left\|\mathbf{H}_{\mathrm{k}}^{-1} \mathbf{H}_{\mathrm{s}}^{H} \mathbf{F}^{H} \mathbf{H}_{\mathrm{y}}^{H}\right\|_{F}^{2} .
\end{aligned}
$$

